JETENSKAP

\title{
Lower estimates for a number of closed trajectories of generalized billiards
}

\author{
Fedor S. Duzhin
}

Stockholm 2005

Doctoral Dissertation

Royal Institute of Technology

Department of Mathematics 
Akademisk avhandling som med tillstånd av Kungl Tekniska Högskolan framlägges till offentlig granskning för avläggande av filosofie doktorsexamen fredagen den 25 mars 2005 Kungl Tekniska Högskolan, Valhallavägen 79, Stockholm.

ISBN 91-7283-993-7

TRITA-MAT-05-MA-04

ISSN 1401-2278

ISRN KTH/MAT/DA 05/02-SE

(C) Fedor S. Duzhin, March 2005

Universitetsservice US AB, Stockholm 2005 


\section{Abstract}

Consider a billiard system on the Euclidean plane. Let $T$ be a strictly convex domain in $\mathbb{R}^{2}$ with a smooth boundary. A billiard ball is a point that moves in $T$ along a straight line and rebounds from the boundary making the angle of incidence equal to the angle of reflection. George Birkhoff considered the following question: given an integer $k$, how to estimate from below the number of closed billiard trajectories of period $k$ ? He proved that if $k>2$, then there are at least $\varphi(k)$ closed trajectories of a billiard ball in $T$ with $k$ rebounds, where $\varphi(k)$ is the number of integers $q<k$ such that $q$ and $k$ are coprime.

In the present thesis G. Birkhoff's problem is studied in the most generality. A definition of a closed trajectory for a billiard whose boundary (in some sense) is an arbitrary manifold embedded in a Euclidean space, is given.

The main result is Theorem 1.2.1 containing the following estimates. Let $M^{m} \subset \mathbb{R}^{n}$ be a smooth closed manifold, $B=\sum_{i=0}^{m} \operatorname{dim} H_{i}\left(M ; \mathbb{Z}_{2}\right)$. If the embedding $M \hookrightarrow \mathbb{R}^{n}$ is generic, then the number of closed billiard trajectories of period 2 is not less than

$$
\frac{B^{2}+(m-1) B}{2},
$$

while the number of closed billiard trajectories of period 3 is at least

$$
\frac{B^{3}+3(m-1) B^{2}+2 B}{6} .
$$

If $p>3$ is a prime integer, then the number of closed billiard trajectories of period $p$ is at least

$$
\frac{(B-1)\left((B-1)^{p-1}-1\right)}{2 p}+\frac{m B}{2}(p-1) .
$$

The last chapter of the thesis ivestigates whether all the obtained esimates are sharp.

2000 Mathematics Subject Classification. Primary: 55N, Secondary: 58K05, 37A 


\section{Acknowledgments}

First of all I would like to express my deepest gratitude to my father Sergei Duzhin for his love and warm encouragement during all my life. He initiated my interest to mathematics, showed me its beauty, so his influence determined my choice to become a mathematician.

I am also glad to thank all my senior advisers: Sabir Gusein-Zade from Moscow State University who helped me to do first steps in the science, Michael Shapiro from Michigan State University who invited me to Sweden and led my research here, Carel Faber from the Royal Institute of Technology who supervised my Ph.D. studies in the final stage.

I thank all my teachers: Jakov Zaidelman and Larisa Gorelysheva from my high school in the city of Pereslavl-Zalessky, Sergei Chmutov and Andrei Nemytykh who together with my father led the mathematical study group for high school students in Pereslavl-Zalessky, Vladimir Dolnikov and Sergei Volchenkov from Yaroslavl Distance Learning School, Igor Chubarov and Mikhail Deriabin from Moscow State University who were not just my teachers, but became friends of mine.

I am thankful to my colleagues for a careful reading of my papers and useful discussions: Levan Alania who was an opponent of my master thesis, Oleg Viro who is an opponent of the doctoral thesis, Peter Pushkar whose research I am continuing in the present work, and, especially, Michael Faber and Sergei Tabachnikov who noticed an error in my paper. Its correction is a perceptible part of the present work.

I am indebted to all the schools I studied at: the gymnasium no. 7 in Pereslavl-Zalessky, the Department of Mechanics and Mathematics, Moscow State University, the Department of Mathematics, the Royal Institute of Technology. I would like to thank personally Irina Vassilieva, the director of Yaroslavl Distance Learning Mathematical School, and Ari Laptev, the proprefect of the Department of Mathematics, Royal Institute of Technology for their friendly engagement. I am also happy to mention here the Russian Foundation of Fundamental Research for financial support and the foundation Knut och Alice Wallenbergs Stifelse for traveling grants that allowed me to visit some conferences. 
Finally, I would like to thank sincerely my friends and colleagues Dmitri Beliaev, Dmitri Apassov, Alexander Romanov, Sergei Shadrin for useful scientific discussions and for their warm attitude towards me.

ISBN 91-7283-993-7 • TRITA-MAT-05-MA-04 • ISSN 1401-2278•ISRN KTH/MAT/DA 05/02-SE 


\section{Contents}

1 Introduction 1

1.1 Brief history of the problem .................. 2

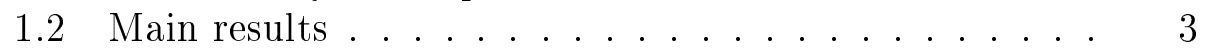

1.3 Ideas and methods .................. 5

1.4 Related questions and conjectures ......... 11

2 Morse theory 13

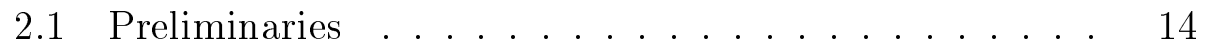

2.2 Estimate by Morse theory . . . . . . . . . . . 16

2.3 Generic embeddings . . . . . . . . . . . 27

3 Homology of symmetric products 31

3.1 A. Dold's theory ..................... 32

3.2 Lemma on symmetric products ......... 36

4 Lower estimates 43

4.1 Period 2 . . . . . . . . . . . . . . 44

4.2 Period $3 \ldots \ldots \ldots . \ldots \ldots$

4.3 Estimate for an arbitrary period ......... 54

5 Sharpness of given estimates $\quad 59$

5.1 Plane billiards ....................... 60

5.2 Some conjecture on torus . . . . . . . . . . 62 
Chapter 1

\section{Introduction}




\subsection{Brief history of the problem}

The mathematical study of periodic billiard trajectories is a classical question and goes back to George Birkhoff. A billiard is a motion of a particle when a field of force is lacking. Trajectories of such particle are geodesics.

Consider a billiard system on the Euclidean plane. Let $T$ be a strictly convex domain in $\mathbb{R}^{2}$ with a smooth boundary $\partial T$. A billiard ball is a point that moves in $T$ along a straight line and rebounds from the boundary making the angle of incidence equal to the angle of reflection.

George Birkhoff stated and solved the following problem in [1]: given an integer $k$, estimate from below the number of closed billiard trajectories such that the billiard ball rebounds from $\partial T$ exactly $k$ times. He proved

Theorem 1.1.1. Suppose $k>2$ is an integer. Then there are at least $\varphi(k)$ closed trajectories of a billiard ball in $T$ with $k$ rebounds, where $\varphi(k)$ is the number if integers $q<k$ such that $q$ and $k$ are coprime.

G. Birkhoff did not investigated whether his estimate was sharp. A positive answer to this question is given by Theorem 5.1.1 in this thesis.

G. Birkhoff's considerations were generalized in two directions. First, it is naturally to increase the dimension of the billiard table. Michael Farber and Sergei Tabachnikov proved a result similar to G. Birkhoff's for the case of larger dimension (see [7] and [8]):

Theorem 1.1.2. Let $T$ be a strictly convex domain in $\mathbb{R}^{m+1}$ with a smooth boundary $M$ that topologically is an $m$-sphere $S^{m}, k$ an odd integer. If $m \geq 2$, then the number of distinct closed billiard trajectories in $T$ with $k$ rebounds is not less than

$$
\log _{2}(k-1)+m .
$$

If the embedding $M \approx S^{m} \rightarrow \mathbb{R}^{m+1}$ is generic, then the number of closed billiard trajectories in $T$ with $k$ rebounds (for any $m$ ) is not less than

$$
m(k-1) .
$$


Here generic embeddings form an open everywhere dense set in the space of all smooth embeddings $M \hookrightarrow \mathbb{R}^{n}$. The exact definition is given below.

Let us mark that if $k=a b$ is not prime, then this theorem counts not only pure billiard trajectories of period $k$, but also trajectories of period $a$ repeated $b$ times. Therefore one considers usually only billiard trajectories with a prime number of rebounds.

On the other hand, one may look at very general billiard tables. Actually a billiard ball may rebound from not necessarily a topological sphere, but from an arbitrary manifold. Consider for example a closed billiard trajectory of period 2. In fact, it is a line segment orthogonal to a billiard table at both its endpoints. One may study such double normals even if a billiard table's boundary is not a hypersurface, but some smooth closed submanifold of a Euclidean space. P. Pushkar found an estimate for the number of double normals of an arbitrary smooth manifold immersed in a Euclidean space. Namely he proved in [6] the following fact:

Let $M$ be a smooth closed connected $m$-dimensional manifold immersed in the Euclidean space $\mathbb{R}^{n}, A$ and $B$ be points of $M$. A line segment $A B$ is a double normal if $A B$ is orthogonal to both tangent spaces $T_{A}(M)$ and $T_{B}(M)$.

Theorem 1.1.3. Suppose $B=\sum_{i=0}^{m} \operatorname{dim} H_{i}\left(M ; \mathbb{Z}_{2}\right)$. If the immersion $M \rightarrow \mathbb{R}^{n}$ is generic, then the number of double normals is not less than

$$
\frac{B^{2}+(m-1) B}{2}
$$

In paper [9] P. Pushkar's results were generalized by myself for closed billiard trajectories of period 3. Notice that our methods actually give an estimate only for embeddings.

Now proceed by our own results.

\subsection{Main results}

First let us give a general definition of a closed billiard trajectory for an arbitrary manifold immersed into a Euclidean space. Suppose $e$ : $M^{m} \rightarrow \mathbb{R}^{n}$ is an immersion. 
Definition 1.2.1. An ordered set $\left(x_{1}, \ldots, x_{k}\right) \in M \times \cdots \times M$ is called a periodic (closed) billiard trajectory if the following conditions hold for each cyclic index $i \in \mathbb{Z}_{k}$ (we mean $i=i+k$ ):

1. $e\left(x_{i}\right) \neq e\left(x_{i+1}\right)$,

2. $\frac{e\left(x_{i}\right)-e\left(x_{i+1}\right)}{\left\|e\left(x_{i}\right)-e\left(x_{i+1}\right)\right\|}+\frac{e\left(x_{i}\right)-e\left(x_{i-1}\right)}{\left\|e\left(x_{i}\right)-e\left(i_{i-1}\right)\right\|} \perp T_{e\left(x_{i}\right)} e(M)$.

Clearly, in the case of codimension 1 the second condition means that the angle of incidence equals to the angle of reflection, but the present definition is also valid when $M$ is an immersed into $\mathbb{R}^{n}$ manifold of an arbitrary codimension.

The main result of the present work is

Theorem 1.2.1. Let $M$ be a smooth closed connected m-dimensional manifold embedded in the Euclidean space $\mathbb{R}^{n}, B=\sum_{i=0}^{m} \operatorname{dim} H_{i}\left(M ; \mathbb{Z}_{2}\right)$ the sum of Betti numbers. Suppose the embedding $M \rightarrow \mathbb{R}^{n}$ is generic, $p>3$ is a prime integer. Then the number of p-periodic billiard trajectories in $M$ is not less than

$$
\frac{(B-1)\left((B-1)^{p-1}-1\right)}{2 p}+\frac{m B}{2}(p-1) .
$$

If $p=3$, then this estimate can be strengthened. The number of 3periodic billiard trajectories for a generic embedding $M^{m} \rightarrow \mathbb{R}^{n}$ is at least

$$
\frac{B^{3}+3(m-1) B^{2}+2 B}{6}
$$

Finally, for $p=2$ our methods give the same estimate as P. Pushkar's. The number of double normals is at least

$$
\frac{B^{2}+(m-1) B}{2} \text {. }
$$

As we mentioned before, George Birkhoff did not prove that his estimate was sharp. Our Theorem 5.1.1 from Chapter 5 gives a positive answer to this question. Namely it says that for any prime $p$ there exists an embedding $S^{1} \hookrightarrow \mathbb{R}^{2}$ such that there are exactly $p-1$ closed billiard trajectories of period $p$. 


\subsection{Ideas and methods}

Let us recall that the original problem by G. Birkhoff is to estimate the number of closed billiard trajectories of period $k$ in a strictly convex domain $T \subset \mathbb{R}^{2}$ bounded by the smooth curve, say $C=\partial T$. G. Birkhoff's idea is to apply the geometric theorem of H. Poincaré as follows.

A billiard motion is defined by the initial point and by the initial direction. Let $P$ be a point on the curve $C=\partial T, \vec{v}$ a unit vector directed inwards the domain $T$. The curve $C$ is an image of the inclusion $e: S^{1} \rightarrow \mathbb{R}^{2}$. Put $\varphi=e^{-1}(P)$ and by $\theta$ denote the angle between $\vec{v}$ and the positive direction of the tangent at the point $P$. Thus $\theta$ varies from 0 to $\pi$. The set of all possible couples $(\varphi, \theta)$ is an annulus in polar coordinates $(\varphi, r=\pi+\theta)$.

The geometric theorem of $\mathrm{H}$. Poincaré claims that if $R$ is a oneto-one continuous area-preserving mapping of an annulus to itself such that the inner and the outer circles are invariant and $R$ rotates them to different directions, then there exist at least two points of the annulus invariant under the mapping $R$.

The annulus of all billiard trajectories is constructed above. Let us describe a corresponding transformation $R$ of the annulus. Consider a point $P \in C$ and a unit vector $\vec{v}$. Let $P^{\prime} \neq P$ be a point of the curve $C$ such that $\overrightarrow{P P^{\prime}} \| \vec{v}$ that is a next vertex of the corresponding billiard trajectory and let $\overrightarrow{v^{\prime}}$ be a direction after the reflection at the point $P^{\prime}$. Put $R(P, \vec{v})=\left(P^{\prime}, \overrightarrow{v^{\prime}}\right)$. Then the transformation $R$ is areapreserving, where the area of a domain $Z$ is equal to the double integral $\iint \sin \theta d \theta d \varphi$ taken over $Z$. It is not very easy to prove this areapreserving property, so for the details see [1].

Notice that $R$ actually is identical on the inner and on the outer circles of the annulus. Nevertheless, if we look to a circle $S_{\theta}=\{r=$ $\pi+\theta$ \} with $\theta$ varying from 0 to $\pi$, then we see that $R$ effects one complete rotation of the circle $S_{\theta}$. In fact, $R\left(S_{\pi}\right)$ is the circle $S_{\pi}$ that has undergone a single complete rotation. Radial segments $\varphi=$ const are taken by $R$ into curves starting and ending at the same points $r=\pi$ and $r=2 \pi$, but twirling once around the ring.

Then $k$-periodic billiard trajectories correspond to invariant points of the transformation $R^{k}$. As we mentioned before, $R$ fixes the inner circle of the annulus and rotates the outer one in the positive direction. Thus $R^{k}$ fixes the inner circle and rotates the outer circle $k$ times in the 
positive direction. Since an angle is defined up to an additive constant $2 \pi i$, we can say that $R^{k}$ rotates the inner circle $i$ times to negative direction and effects $k-i$ complete rotations of the outer circle in the positive direction and therefore the Poincaré theorem is applicable. It is possible to extend Poincaré's theorem and prove that there exist at least two such invariant points of each rotation number (if $P_{1} \ldots P_{k}$ is a closed polygon, then the rotation number is equal to the sum of all the angles between $\overrightarrow{P_{i-1} P_{i}}$ and $\overrightarrow{P_{i} P_{i+1}}$ divided by $2 \pi$ ). The possible rotation numbers are those coprime with $k$, at most $\frac{k}{2}$. This proves G. Birkhoff's estimate. Notice that if $k^{\prime}$ and $k$ have a common divisor $a>1$, then $k$-periodic billiard trajectories of rotation number $k^{\prime}$ may happen to be invariant points of the mapping $T^{a}$, that's why we consider only rotation numbers coprime with $k$.

Another approach is to consider the function $l:\left(S^{1}\right)^{\times k} \rightarrow \mathbb{R}$ equal to the length of a closed polygon $P_{1} \ldots P_{k}$ (all of $P_{i}$ lie on the curve $C$ ). It is not hard to see that critical points of this function are exactly closed billiard trajectories of period $k$ and therefore it is naturally to apply Morse and Lyusternik-Shnirelman theories to investigate closed billiard trajectories. Morse theory gives sharper estimates, but requires some additional condition: the function $l$ has to be generic in some sense. In spite of Birkhoff's method has some advantages (critical points may be degenerate and the period $k$ is not necessarily prime), it is absolutely unapplicable to billiard motion in $\mathbb{R}^{m}, m>2$. Indeed, there does not exist any natural equivalent either to Poincaré's theorem or to the notion of rotation number in multi-dimensional Euclidean space.

The simplest case to apply Morse theory is $k=2$. Suppose we deal with a convex body $T \subset \mathbb{R}^{n}$, its boundary $\partial T$ is a smooth hypersurface that topologically is a sphere $S^{n-1}$. As we mentioned before, a closed billiard trajectory of period 2 is a line segment orthogonal to a given manifold at both its endpoints. The distance function is $l=\|x-y\|$ : $S^{n-1} \times S^{n-1} \rightarrow \mathbb{R}$, double normals are exactly its critical points outside the diagonal $\Delta=\{(x, x)\} \subset S^{n-1} \times S^{n-1}$, we suppose that all of them are non-degenerate. There is a symmetry here: the function $l$ is invariant under the involution $(x, y) \rightarrow(y, x)$, thus $l$ is actually defined on the symmetric square $\frac{S^{n-1} \times S^{n-1}}{(x, y)=(y, x)}$ - indeed, $P Q$ and $Q P$ are the same line segment. We see that our problem therefore is to estimate 
the number of critical points of the function

$$
l: \frac{S^{n-1} \times S^{n-1}}{(x, y)=(y, x)} \rightarrow \mathbb{R}
$$

Since the space $\frac{S^{n-1} \times S^{n-1}}{(x, y)=(y, x)}$ is not smooth, we cannot apply Morse inequalities directly. Nevertheless, it is smooth outside the diagonal $\Delta=\{(x, y): l(x, y)=0\}$ and this fact allows us to construct a chain complex using the function $l$ in the same way as one uses Morse function on a smooth manifold. Morse inequalities in this situation imply that the number of double normals is at least

$$
\sum \operatorname{dim} H_{q}\left(\frac{S^{n-1} \times S^{n-1}}{(x, y)=(y, x)}, \Delta ; \mathbb{Z}_{2}\right) .
$$

It is not hard to calculate this sum and we do it later, in Chapter 4. The sum equals $n$. This estimate is sharp: for an ellipsoid with all different axes the number of double normals is exactly $n$ - the double normals are its axes. This estimate was found by M. Morse himself in $[2]$.

N. H. Kuiper proved it in [3] for non-generic convex bodies in $\mathbb{R}^{n}$. His idea is as follows. Let $d$ be a direction in the space $\mathbb{R}^{n}$ that is a point of the projective space $\mathbb{R} P^{n-1}$. Instead of considering the function $l$ defined on the Cartesian square $S^{n-1} \times S^{n-1}$ we consider a function

$$
\tilde{l}(d)=\sup _{x, y \in T, \overrightarrow{x y} \| d} l(x, y)
$$

defined on the projective space $\mathbb{R} P^{n-1}$. Kupier proved that critical points of the function $\tilde{l}$ are exactly directions parallel to double normals. Thus their number is estimated by the cohomological length of $\mathbb{R} P^{n-1}$.

The definition of a double normal makes sense when we replace the sphere $S^{n-1}$ with an arbitrary $m$-dimensional manifold $M \subset \mathbb{R}^{n}$. F. Takens and J. White in [4] applied Morse theory in this more general case and proved that the number of double normals for a generic smooth closed manifold embedded to a Euclidean space is at least

$$
2 \sum_{i=0}^{2 m}\left[\sum_{j=0}^{i} \frac{(-1)^{j}}{2} \sum_{k=1}^{i-j} b_{k} b_{i-j-k}\right]-\left[\sum_{j=0}^{2 m} \frac{(-1)^{j}}{2} \sum_{k=1}^{2 m-j} b_{k} b_{2 m-j-k}\right],
$$

where $b_{q}=\operatorname{dim} H_{q}\left(M ; \mathbb{Z}_{2}\right),[a]$ denotes the largest integer not exceeding $a$. Takens and White did not involve the natural $\mathbb{Z}_{2}$-symmetry, their 
estimate is an application of Morse inequalities directly to the Cartesian square $M \times M$, in fact, they used a modified version of 3.1 :

$$
\frac{1}{2} \sum \operatorname{dim} H_{q}\left(S^{n-1} \times S^{n-1}, \Delta ; \mathbb{Z}_{2}\right) .
$$

That's why their estimate is not sharp and P. Pushkar succeeded to strengthen it in [6].

Pushkar introduced another function whose critical points correspond to double normals. Namely suppose $M^{m} \subset \mathbb{R}^{n}$. Let $S^{n-1}=$ $\left\{\left(u_{1}, \ldots, u_{n}\right): u_{1}^{2}+\cdots+u_{n}^{2}=1\right\} \subset \mathbb{R}^{n}$. Put

$$
f(a, x, y)=\langle a, x-y\rangle: S^{n-1} \times M \times M \rightarrow \mathbb{R},
$$

where $a \in S^{n-1}, x, y \in M$. It is not hard to see that $(x, y)$ is a double normal whenever $\left(\frac{x-y}{\|x-y\|}, x, y\right)$ is a critical point of the function $f$. There is a symmetry here: the function $f$ is invariant under the involution $s:(a, x, y) \rightarrow(-a, y, x)$. This involution has no fixed points and thus the quotient $S^{n-1} \times M \times M / s$ is a smooth manifold. In order to apply Morse inequalities one should calculate the homology groups $H_{*}\left(S^{n-1} \times M \times M / s\right)$. Pushkar showed that the homology $H_{*}\left(S^{n-1} \times\right.$ $M \times M / s)$ is determined by the homology $H_{*}(M)$ and therefore we may replace the manifold $M$ with a bouquet of spheres $X$ such that $H_{*}\left(X ; \mathbb{Z}_{2}\right) \equiv H_{*}\left(M ; \mathbb{Z}_{2}\right)$. Using all this machinery he proved that the number of double normals is at least

$$
\frac{B^{2}+(m-1) B}{2}
$$

where $B=\sum \operatorname{dim} H_{q}\left(M ; \mathbb{Z}_{2}\right)$. Pushkar's estimate is sharp for a wide class of manifolds: two-dimensional orientable surfaces, spheres, products of spheres.

Pushkar's approach has a certain advantage - his theorem holds for immersions, while our method applied to immersions counts degenerate billiard trajectories (for example, transversal self-intersections that are double normals of zero length) among all. Nevertheless, our attempt to construct a function similar to Pushkar's one whose critical points correspond to closed billiard trajectories of an arbitrary period has failed. Indeed, a natural function

$$
f_{k}=\sum_{i \in \mathbb{Z}_{k}}\left\langle a_{i}, x_{i}-x_{i+1}\right\rangle:\left(S^{n-1}\right)^{\times k} \times M^{\times k} \rightarrow \mathbb{R}, k \geq 3
$$


(as above, we mean $i=i+k$ ), has not only closed billiard trajectories as critical points and therefore it cannot help us to get a lower bound for the number of periodic billiard trajectories. Notice that we consider this function in more details in Section 2.2.

Another natural way to generalize Pushkar's function is

$$
g_{k}=\langle A, P\rangle: S^{n k-1} \times M^{\times k} \rightarrow \mathbb{R},
$$

where $P$ is an ordered set $\left(x_{2}-x_{1}, x_{3}-x_{2}, \ldots, x_{k}-x_{k-1}, x_{1}-x_{k}\right)$. Here each component $x_{i}-x_{i-1}$ belongs to the $n$-dimensional vector space $\mathbb{R}^{n} \supset M$ and thus $P \in \mathbb{R}^{n k}, A \in S^{n k-1} \subset \mathbb{R}^{n k}$. Since $A$ and $P$ are both the points of the Euclidean space $\mathbb{R}^{n k}$, their inner product $g_{k}=\langle A, P\rangle$ is well-defined. Let us recall that closed billiard trajectories are critical points of the function

$l=\left\|x_{1}-x_{2}\right\|+\left\|x_{2}-x_{3}\right\|+\cdots+\left\|x_{k-1}-x_{k}\right\|+\left\|x_{k}-x_{1}\right\|: M^{\times k} \rightarrow \mathbb{R}$.

Introduce the following

Definition 1.3.1. An ordered set $\left(x_{1}, \ldots, x_{k}\right) \in M \times \cdots \times M$ is called $a$ periodic (closed) quadratic billiard trajectory if the following conditions hold for each cyclic index $i \in \mathbb{Z}_{k}$ (we mean $i=i+k$ ):

1. $x_{i} \neq x_{i+1}$,

2. $\left(x_{i}-x_{i+1}\right)+\left(x_{i}-x_{i-1}\right) \perp T_{x_{i}} M$.

Then such quadratic billiard trajectories are critical points of the function

$\tilde{l}=\left\|x_{1}-x_{2}\right\|^{2}+\left\|x_{2}-x_{3}\right\|^{2}+\cdots+\left\|x_{k-1}-x_{k}\right\|^{2}+\left\|x_{k}-x_{1}\right\|^{2}: M^{\times k} \rightarrow \mathbb{R}$.

Now it is easy to see that critical points of the function $g_{k}$ correspond to quadratic billiard trajectories. Studying these quadratic billiards is a natural and sensible problem, but it lies beyond the main subject of our research.

As far as we know, the first attempt to extend Birkhoff's considerations and results to multi-dimensional billiards is the paper [5] by I. Babenko. Unfortunately, it contains an error and only M. Farber and S. Tabachnikov in [7] and [8] proved a theorem similar to Birkhoff's. They studied closed billiard trajectories of an odd period $k$ in a convex domain $T \subset \mathbb{R}^{m+1}$ whose boundary $\partial T$ topologically is the sphere 
$S^{m}$. Their approach involves application of Morse and LyusternikShnirelman theories directly to the function

$l=\left\|x_{1}-x_{2}\right\|+\left\|x_{2}-x_{3}\right\|+\cdots+\left\|x_{k-1}-x_{k}\right\|+\left\|x_{k}-x_{1}\right\|:\left(S^{m}\right)^{\times k} \rightarrow \mathbb{R}$.

They proved that the number of $k$-periodic trajectories can be estimated in terms of the cohomology ring $H_{*}\left(\left(S^{m}\right)^{\times k} / D_{k}, \Delta\right)$. Here the dihedral group $D_{k}$ acts on the Cartesian degree $\left(S^{m}\right)^{\times k}$ by the cyclic permutation

$$
\left(x_{1}, x_{2}, \ldots, x_{k}\right) \rightarrow\left(x_{2}, x_{3}, \ldots, x_{k}, x_{1}\right)
$$

and the reflection

$$
\left(x_{1}, x_{2}, \ldots, x_{k}\right) \rightarrow\left(x_{k}, x_{k-1}, \ldots, x_{1}\right) .
$$

By $\Delta$ we denote the subset of the $k$ th Cartesian degree $\left(S^{m}\right)^{\times k}$ consisting of all the points $\left(x_{1}, \ldots, x_{k}\right)$ such that $x_{i}=x_{i+1}$ for some $i$ or $x_{k}=x_{1}$. Then the length of this cohomology ring estimates the number of closed billiard trajectories while its Betti numbers sum is a lower bound for this number in a generic situation. Farber and Tabachnikov construct a spectral sequence converging to the cohomology $\left.H_{*}\left(S^{m}\right)^{\times k}, \Delta\right)$ and then study how the dihedral group $D_{k}$ acts on it. The whole calculation is very technical and complicated. The result is $\log _{2}(k-1)+m$ for arbitrary domains and $m(k-1)$ for generic ones.

We consider a more general problem. We deal with a generic embedding $e: M^{m} \rightarrow \mathbb{R}^{n}$ and estimate the number of closed billiard trajectories of a certain period $p^{1}$. The idea of the proof is as follows. In Chapter 2 we apply Morse theory to the object being studied and show that corresponding Morse inequalities involve the homology

$$
H_{*}\left(M^{\times k} / D_{k}, \Delta\right) .
$$

They imply that the number of closed billiard trajectories of period $k$ is at least

$$
\sum \operatorname{dim} H_{q}\left(M^{\times k} / D_{k}, \Delta ; \mathbb{Z}_{2}\right) .
$$

Thus our goal is to calculate the sum 3.4. In Chapter 3.2 we prove that if two spaces have isomorphic homology groups, then the homology groups 3.3 are isomorphic as well. Thus it is enough to calculate the

\footnotetext{
${ }^{1}$ For the time present we are able to prove an estimate only for prime periods
} 
expression 3.4 for a bouquet of spheres. Indeed, if $b_{q}=\operatorname{dim} H_{q}\left(M ; \mathbb{Z}_{2}\right)$, $q=0,1 \ldots, m$, then the bouquet

$$
X=S^{m} \vee S_{1}^{m-1} \vee \cdots \vee S_{b_{m-1}}^{m-1} \vee \cdots \vee S_{1}^{1} \vee \cdots \vee S_{b_{1}}^{1}
$$

has the same $\mathbb{Z}_{2}$-homology groups as the manifold $M$ and hence the sum 3.4 for $X$ equals the sum 3.4 for $M$. In Chapter 4 we calculate this sum.

\subsection{Related questions and conjectures}

Consider now the situation from the more general point of view according to V. Arnold (see [10]). We have dealt until now with generic (or arbitrary) embeddings (or immersions) of a certain manifold to a Euclidean space of an arbitrary dimension. We could investigate a narrower class of mappings. Suppose a manifold $M$ and an integer $n$ are fixed and $\Omega$ is a functional space of smooth mappings $M \rightarrow \mathbb{R}^{n}$. There is a subset $\Omega_{0} \subset \Omega$ called a discriminant, while all the mappings $f \notin \Omega_{0}$ are appropriate. We demand that all non-immersions belong to the discriminant. In such a situation a function $I$ defined on the set of appropriate mappings $\Omega \backslash \Omega_{0}$ is an invariant if $I$ is constant on connected components.

For example, if $M=S^{1}, n=3$, and appropriate mappings are embeddings, then our appropriate object is a knot. If $M=S^{1}, n=2$, and appropriate mappings are immersions such that the only singularities are simple double points (no triple points and self-tangent points are allowed), then we deal with closed plane curves. Knots and plane curves are very classical objects to study.

Now let the space of mappings $\Omega$ and the discriminant $\Omega_{0}$ be fixed. Then the minimal number of closed billiard trajectories for all generic immersions belonging to a given connected component of $\Omega \backslash \Omega_{0}$ is an invariant. It seems to be a new one for both knots and plane curves. A very natural problem is to estimate (or may be even to express if possible) it in terms of known invariants. Consider, for example, a plane curve that is an immersion $e: S^{1} \rightarrow \mathbb{R}^{2}$. The simplest invariant is a number of self-intersections $d$. Takens and White proved in [4] the following

Theorem 1.4.1. If the immersion $e: S^{1} \rightarrow \mathbb{R}^{2}$ is generic, then the number of double normals is at least $2+d$. 
Their idea is to modify 3.1. Actually they proved that if $e: M \rightarrow \mathbb{R}^{n}$ is a generic immersion, then the number of double normals is bounded from below by

$$
\frac{1}{2} \sum \operatorname{dim} H_{q}\left(M \times M, \Delta \cup \Delta_{e} ; \mathbb{Z}_{2}\right),
$$

where $\Delta=\{(x, x)\} \subset M \times M$ and $\Delta=\{(x, y): e(x)=e(y)\} \subset$ $M \times M$. If $M=S^{1}$ is immersed to $\mathbb{R}^{2}$, then $\Delta_{e}$ is the set of all the self-intersection points. The summand $d$ in the last theorem occurs from adding $\Delta_{e}$ to our considerations.

In fact, one may define and investigate closed billiard trajectories when dealing not necessarily with a smooth manifold, but with some other object, like P. Pushkar who in [6] studied double normals of wave fronts, but this topic lies beyond our research. 
Chapter 2

Morse theory 


\subsection{Preliminaries}

Let $M$ be a smooth closed connected $m$-dimensional manifold embedded in the Euclidean space $\mathbb{R}^{n}$ (clearly, $m<n$ ). Let us recall a main

Definition 2.1.1. An ordered set $\left(x_{1}, \ldots, x_{k}\right) \in M \times \cdots \times M$ is called a periodic (closed) billiard trajectory if the following conditions hold for each cyclic index $i \in \mathbb{Z}_{k}$ (we mean $i=i+k$ ):

1. $x_{i} \neq x_{i+1}$,

2. $\frac{x_{i}-x_{i+1}}{\left\|x_{i}-x_{i+1}\right\|}+\frac{x_{i}-x_{i-1}}{\left\|x_{i}-x_{i-1}\right\|} \perp T_{x_{i}} M$.

Let us notice that if such an orbit is given, then it can be considered started at any of its vertices and the direction may be reversed. We formalize this remark as follows. The dihedral group $D_{k}$ acts on the set of closed billiard trajectories. This action is generated by the cyclic permutation

$$
\left(x_{1}, x_{2}, \ldots, x_{k}\right) \rightarrow\left(x_{2}, x_{3}, \ldots, x_{k}, x_{1}\right)
$$

and the reflection

$$
\left(x_{1}, x_{2}, \ldots, x_{k}\right) \rightarrow\left(x_{k}, x_{k-1}, \ldots, x_{1}\right) .
$$

Orbits of this action are also called periodic (closed) billiard trajectories. In fact, that are the orbits are being studied and estimated here (and in $[1],[7],[8],[6],[9]$ as well).

Let us introduce some notations:

$$
M^{\times k}=M \times \cdots \times M
$$

is the $k$ th Cartesian power of the space $M$,

$$
\Delta^{(j)}=\bigcup_{i \in \mathbb{Z}_{k}}\left\{\left(x_{1}, \ldots, x_{k}\right): x_{i}=x_{i+1}=\cdots=x_{i+k-j-1}\right\} \subset M^{\times k}
$$

is the $j$ th diagonal of $M^{\times k}$. Clearly, we have

$$
\{(x, x, \ldots, x)\}=\Delta^{(0)} \subset \Delta^{(1)} \subset \cdots \subset \Delta^{(k-2)} \subset \Delta^{(k-1)}=M^{\times k} .
$$

By $\Delta$ we denote $\Delta^{(k-2)}=\bigcup_{i \in \mathbb{Z}_{k}}\left\{x_{i}=x_{i+1}\right\} \subset M^{\times k}$. Let

$$
l_{k}=\sum_{i \in \mathbb{Z}_{k}}\left\|x_{i}-x_{i+1}\right\|: M^{\times k} \rightarrow \mathbb{R}
$$

be the length function of a closed polygon, where |||| is the norm of the ambient space $\mathbb{R}^{n}$. The function $l_{k}$ is smooth outside of the diagonal 
$\Delta$. Moreover, $\Delta$ is the set of all the points where $l_{k}$ is not smooth. If the value of $k$ is fixed, then by $l$ without an index we mean the function $l_{k}$.

The symmetric group $S_{k}$ acts on $M^{\times k}$ by permuting the factors, and so does the dihedral group $D_{k} \subset S_{k}$. The function $l$ is invariant under this last action and therefore induces the function on the quotient space $M^{\times k} / D_{k}$. Let us denote the quotient space $\Delta / D_{k}$ and the induced function $M^{\times k} / D_{k} \rightarrow \mathbb{R}$ by the same letters $\Delta$ and $l$. We hope it does not cause any misunderstanding in further. Similarly, $\Delta^{(k)}$ denotes also the quotient under the action of the dihedral group $D_{k}$.

The following fact is well known, but let us prove it anyway:

Lemma 2.1.1. Let $M$ be a smooth manifold embedded in $\mathbb{R}^{n}$. Then $k$-periodic billiard trajectories are exactly critical points of the function $l$ outside of $\Delta$.

Proof. Differentiating $l=\sum_{i \in \mathbb{Z}_{k}}\left\|x_{i}-x_{i+1}\right\|$ with respect to $x_{i}$, we obtain

$$
\partial_{x_{i}} l=\frac{x_{i}-x_{i+1}}{\left\|x_{i}-x_{i+1}\right\|}+\frac{x_{i}-x_{i-1}}{\left\|x_{i}-x_{i-1}\right\|} .
$$

The point $\left(x_{1}, \ldots, x_{k}\right)$ is a critical point of the function $l$ if and only if all $\partial_{x_{i}} l$ are orthogonal to the manifold $M$.

Definition 2.1.2. We say that an embedding $M \hookrightarrow \mathbb{R}^{n}$ is generic (or, more exact, $k$-generic) if all the isolated critical points of all of the length functions $l_{k^{\prime}}$ for $k^{\prime} \leq k$ outside the corresponding diagonal $\Delta_{k^{\prime}} \subset M^{\times k^{\prime}}$ are non-degenerate.

Remark 2.1.1. This definition makes sense for immersions as well.

Notice that if $k=a b$ is not prime, then the set of $k$-periodic billiard trajectories includes closed trajectories of period $a$ repeated $b$ times:

$$
\left(x_{1}, x_{2}, \ldots, x_{a}, x_{1}, \ldots, x_{a}, \ldots, x_{1}, \ldots, x_{a}\right) .
$$

That's why in the further chapters we consider only the case $k=p$ is prime. Thus our problem is to estimate the minimal number $B T_{p}(M)$ of critical points of the function $l_{p}$ for all generic embeddings of the manifold $M$ in a Euclidean space. 


\subsection{Estimate by Morse theory}

Let us begin this section with the main lemma followed by all the statements we use in the proof.

Lemma 2.2.1. Let $M$ be a smooth closed connected m-dimensional manifold, $k$ an integer. The minimal number of $k$-periodic billiard trajectories for all generic embeddings of the manifold $M$ in a Euclidean space satisfies

$$
B T_{k}(M) \geq \sum_{q=0}^{m k} \operatorname{dim} H_{q}\left(M^{\times k} / D_{k}, \Delta ; \mathbb{Z}_{2}\right) .
$$

P r o of. This lemma is a direct corollary of the following lemmas 2.2.5 and 2.2.9. Indeed, suppose we have a generic embedding $e: M \rightarrow \mathbb{R}^{n}$. Then $\mathbb{R}^{n} \subset \mathbb{R}^{n+1}$. Obviously, $\mathbb{R}^{n}$ can be deformed a little such that it becomes a strictly convex hypersurface. According to lemma 2.2.9, the number of closed billiard trajectories remains the same. Now lemma 2.2.5 is applicable.

Lemma 2.2.2. Let $M^{m} \subset \mathbb{R}^{n}$ be a smooth closed manifold. Then there exists $\varepsilon_{0}>0$ such that the following condition holds for any point $x_{1} \in M$ :

Let $x_{2}(t), \ldots, x_{k}(t)$ be geodesics starting at the point $x_{1}, \rho_{i}(t)=$ $\left\|x_{i}-x_{i+1}\right\|, \rho_{k}(t)=\left\|x_{k}-x_{1}\right\|$, the function $l$ is defined as usual:

$$
l(t)=\sum_{i=1}^{k} \rho_{k}(t) .
$$

If $\left\|x_{1}-x_{i}\right\|<\varepsilon_{0}$ for each $i=2, \ldots, k$, then for $t>0$

1. all $\rho_{i}^{\prime}(t) \geq 0$ and therefore $l^{\prime}(t)>0$,

2. $\rho_{1}^{\prime}(t)+\cdots+\rho_{k-1}^{\prime}(t) \geq \rho_{k}^{\prime}(t)$.

Proof. Since $M$ is compact, it is sufficient to find such $\varepsilon_{0}$ for any fixed $x \in M$. Now if $x \in M$ is given, then $t=0$ is the local minimum of all the functions $\rho_{i}(t)$ and the strict local minimum of the function $l(t)$. By the triangle inequality, $\rho_{1}+\cdots+\rho_{k-1} \geq \rho_{k}$ and hence $t=0$ is the local minimum of the function $\rho_{1}+\cdots+\rho_{k-1}-\rho_{k}$. The lemma is proved. 
Definition 2.2.1. Suppose $H$ is a smooth surface in the Euclidean space $\mathbb{R}^{n}$. We say that $H$ is strictly convex if for any point $x \in H$ the intersection $T_{x} H \cap H$ consists only of the point $x$ itself. Note that if $H$ is a hypersurface, then this condition means that $H$ is a part of a boundary of a strictly convex domain, i.e., for any couple of points $x, y \in H$ the open line segment $(x, y)$ lies inside the domain.

Lemma 2.2.3. Suppose $M^{m} \subset \mathbb{R}^{n}$ is a smooth closed strictly convex $m$-dimensional manifold. Then for every $\varepsilon>0$ there exists $\delta>0$ such that the following condition holds: if points $x, y, z \in M$ satisfy

1. $x \neq y, y \neq z$,

2. $\|x-z\|>\varepsilon$,

3. $\frac{x-y}{\|x-y\|}-\frac{y-z}{\|y-z\|} \perp T_{y} M$,

then $\|x-y\|>\delta$ and $\|y-z\|>\delta$.

Proof. Suppose the opposite: we have found $\varepsilon_{0}>0$ such that for every $\delta>0$ there exist points $x, y, z \in M$ satisfying

1. $x \neq y, y \neq z$,

2. $\|x-z\|>\varepsilon_{0}$,

3. $\frac{x-y}{\|x-y\|}-\frac{y-z}{\|y-z\|} \perp T_{y} M$,

4. $\|x-y\|<\delta$.

By $x_{j}, y_{j}, z_{j}$ denote the points corresponding to $\delta=\frac{1}{j}$. Since $M$ and the sphere $S^{n-1}$ are compact manifolds, we may assume that there exist limits

$$
\begin{gathered}
x_{j}, y_{j} \rightarrow x_{0}, z_{j} \rightarrow z_{0}, \\
\frac{x_{j}-y_{j}}{\left\|x_{j}-y_{j}\right\|} \rightarrow \vec{v} \in T_{x_{0}} M .
\end{gathered}
$$

Now we obviously have

$$
\vec{v}-\frac{x_{0}-z_{0}}{\left\|x_{0}-z_{0}\right\|} \perp T_{x_{0}} M
$$


Let $\vec{e}, \ldots, \vec{e}_{m}$ be an orthonormal basis for the space $T_{x_{0}} M$ and $\vec{e}_{m+1}, \ldots, \vec{e}_{n}$ an orthonormal basis for $\left(T_{x_{0}} M\right)^{\perp}$. Suppose

$$
\begin{gathered}
\vec{v}=\sum_{i=1}^{m} a_{i} \vec{e}_{i}, \\
\vec{v}-\frac{x_{0}-z_{0}}{\left\|x_{0}-z_{0}\right\|}=-\sum_{i=m+1}^{n} a_{i} \vec{e}_{i} .
\end{gathered}
$$

Then

$$
\frac{x_{0}-z_{0}}{\left\|x_{0}-z_{0}\right\|}=\sum_{i=1}^{n} a_{i} \vec{e}_{i} .
$$

Calculating the norms we obtain

$$
1=\left\|\frac{x_{0}-z_{0}}{\left\|x_{0}-z_{0}\right\|}\right\|^{2}=\sum_{i=1}^{n} a_{i}^{2} .
$$

On the other hand,

$$
1=\|\vec{v}\|^{2}=\sum_{i=1}^{m} a_{i}^{2} .
$$

Subtracting one from another we get $a_{m+1}=\cdots=a_{n}=0$. Hence $\frac{x_{0}-z_{0}}{\left\|x_{0}-z_{0}\right\|}=\vec{v} \in T_{x_{0}} M$ that is a contradiction to the strict convexity of $H$. $\square$

Lemma 2.2.4. Suppose $M^{m} \subset \mathbb{R}^{n}$ is a smooth closed strictly convex $m$-dimensional manifold. Let $j \geq 3$ be an integer. Then for any $\varepsilon>0$ there exists $\delta>0$ such that the following condition holds for every couple of points $x_{1}, x_{j} \in M,\left\|x_{1}-x_{j}\right\|>\varepsilon$ :

Let $x_{2}(t), \ldots, x_{j-1}(t)$ be geodesics starting at the point $x_{1}$. Define the function

$$
\tilde{l}(t)=\sum_{i=1}^{j-1}\left\|x_{i}-x_{i+1}\right\| .
$$

Then $\left\|x_{1}-x_{2}(t)\right\|<\delta, \ldots,\left\|x_{1}-x_{j-1}(t)\right\|<\delta, t>0$ implies

$$
\tilde{l}^{\prime}(t)>0 \text {. }
$$


Proof. First let us prove the statement for $j=3$. Chose $\delta$ corresponding to $\frac{\varepsilon}{2}$ in the previous lemma. Then $\left\|x_{1}-x_{2}(t)\right\|<\delta<\frac{\varepsilon}{2}$ and $\left\|x_{1}-x_{3}\right\|>\varepsilon$ give $\left\|x_{2}-x_{3}\right\|>\frac{\varepsilon}{2}$. By the previous lemma, we have $\tilde{l}^{\prime}(t) \neq 0$ if $\left\|x_{1}-x_{2}(t)\right\|<\delta$. Since $t=0$ is a strict local minimum of the function $\tilde{l}$, we obtain $\tilde{l}^{\prime}(t)>0$.

Now suppose $j>3$. Put $\delta$ the same as for $k=3$. Then $\tilde{l}=\| x_{1}-$ $x_{2}\|+\cdots+\| x_{j-1}-x_{j} \|$. By lemma 2.2.2, $\tilde{l}^{\prime} \geq\left(\left\|x_{1}-x_{j-1}\right\|+\left\|x_{j-1}-x_{j}\right\|\right)^{\prime}$ that is positive by the argumentation above. The lemma is proved.

Lemma 2.2.5. Suppose $M^{m} \subset \mathbb{R}^{n}$ is a smooth closed connected strictly convex m-dimensional manifold, where $M^{m} \hookrightarrow \mathbb{R}^{n}$ is a generic embedding. Let $k \geq 2$ be an integer. Then the number of closed billiard trajectories in $M$ of period $k$ is at least

$$
\sum_{q=0}^{m k} \operatorname{dim} H_{q}\left(M^{\times k} / D_{k}, \Delta ; \mathbb{Z}_{2}\right) .
$$

Proof. Let us recall that

$$
\Delta=\left\{\left(x_{1}, \ldots, x_{k}\right) \in M^{\times k} / D_{k}: x_{1}=x_{2}\right\}
$$

is the set where the function

$$
l=\sum_{i \in \mathbb{Z}_{k}}\left\|x_{i}-x_{i+1}\right\|: M^{\times k} / D_{k} \rightarrow \mathbb{R}
$$

is not smooth. We suppose that the embedding $M^{m} \hookrightarrow \mathbb{R}^{n}$ is generic, hence all the critical points of the function $l$ outside of the diagonal $\Delta$ are non-degenerate. If the function $l$ has infinitely many critical points, than the lemma is proved since the infinity is greater than any integer. Thus we may suppose that $l$ has only finitely many critical points.

We construct the function $g$ on $X=M^{\times k} / D_{k}$ such that the following conditions hold:

(A) $g \geq 0$.

(B) $g$ is smooth outside $\Delta$.

(C) Critical points of $g$ are the same as those of $l$. Moreover, for each critical point $a_{i} \notin \Delta$ the functions $l$ and $g$ coincide in a small neighborhood of $a_{i}$. Particularly, it implies that their Morse indices are the same. 
(D) $\Delta=\{g=0\}$.

Then our theorem follows from Morse theory. Indeed, a small neighborhood of $\Delta$ in $X / \Delta$ is contractible, that's why we can construct a cell space using the function $g$ in the same way as any Morse function. Further, we apply Morse inequalities and reduce the relative homology to the absolute:

$$
\tilde{H}_{*}(X / \Delta)=H_{*}(X, \Delta) .
$$

Note that if $k=2$, then the lemma is already proved since we can put $g=l$.

Let $\varphi(t)$ be a smooth function such that

1. $0 \leq \varphi \leq 1$,

2. $\left.\varphi\right|_{(-\infty, 0]} \equiv 0$,

3. $\left.\varphi\right|_{[\varepsilon,+\infty)} \equiv 1$,

4. $\left.\varphi^{\prime}\right|_{(0, \varepsilon)}>0$.

Let us show that the function

$$
g_{\varepsilon}\left(x_{1}, \ldots, x_{k}\right)=l\left(x_{1}, \ldots, x_{k}\right)\left(\prod_{i \in \mathbb{Z}_{k}} \varphi\left(\left\|x_{i}-x_{i+1}\right\|\right)\right)
$$

satisfies the conditions (A)-(D) if $\varepsilon$ is small enough.

Recall that $\Delta^{(k-j)}=\left\{\left(x_{1}, \ldots, x_{1}, x_{j+1}, \ldots, x_{k}\right)\right\} \subset \Delta$. A small neighborhood of $\Delta^{(k-j)}$ is given by the inequalities

$$
\left\|x_{i}-x_{i+1}\right\|<\varepsilon_{j}
$$

where $i=1, \ldots, j-1$ for $j>0$ and $i \in \mathbb{Z}_{k}$ for $j=0$. We have the filtration $\Delta^{(0)} \subset \ldots \subset \Delta^{(k-2)}=\Delta$. First we find $\varepsilon_{0}$ defining the neighborhood $U_{\varepsilon_{0}}^{0}\left(\Delta^{(0)}\right)$ and then proceed by induction finding $\varepsilon_{j}$ to define the neighborhood $U_{\varepsilon_{j}}^{j}\left(\Delta^{(j)}\right)$.

Let us start with the inductive basis $j=0$. Suppose $\varepsilon$ is chosen by Lemma 2.2.2. Put $\varepsilon_{0}=\frac{\varepsilon}{k}$. Then

$$
U_{\varepsilon_{0}}^{0}\left(\Delta^{(0)}\right)=\left\{\left(x_{1}, \ldots, x_{k}\right):\left\|x_{i}-x_{i+1}\right\|<\frac{\varepsilon}{k}, i \in \mathbb{Z}_{k}\right\}
$$

is a small neighborhood of $\Delta^{(0)}$. 
We can obtain any point $\left(x_{1}, x_{2}, \ldots, x_{k}\right)$ in this neighborhood if we fix $x_{1}$, emit $k-1$ geodesics from $x_{1}$, and put points $x_{2}, \ldots, x_{k}$ on these geodesics.

Let $x_{i}(t), i=2, \ldots, k$ be geodesics passing through the point $x_{1}$. Then $l\left(x_{1}, \ldots, x_{k}\right)=l(t), g\left(x_{1}, \ldots, x_{k}\right)=g(t)$. Since $\left\|x_{i}-x_{i+1}\right\|<\frac{\varepsilon}{k}$ for each $i$, we have $\left\|x_{1}-x_{i}\right\|<\varepsilon$ for each $i$, hence Lemma 2.2 .2 is applicable. It implies $l^{\prime}(t)>0$ for $t>0$ and $\frac{d}{d t}\left\|x_{i}(t)-x_{i+1}(t)\right\| \geq 0$ for all $i$. Compute the derivative:

$$
g^{\prime}(t)=\left(\prod_{i \in \mathbb{Z}_{k}} \varphi\left(\left\|x_{i}-x_{i+1}\right\|\right)\right)^{\prime} l(t)+\left(\prod_{i \in \mathbb{Z}_{k}} \varphi\left(\left\|x_{i}-x_{i+1}\right\|\right)\right) l^{\prime}(t) .
$$

The inequalities

$$
\begin{gathered}
\varphi>0, l>0,\left\|x_{i}-x_{i+1}\right\|>0 \\
\varphi^{\prime} \geq 0, l^{\prime}(t)>0, \frac{d}{d t} \|\left(x_{i}(t)-x_{i+1}(t) \| \geq 0\right.
\end{gathered}
$$

imply $g^{\prime}(t)>0$ in the neighborhood $U_{\varepsilon}^{0}\left(\Delta^{(0)}\right)$, so we have found the vector $\vec{V}$ (the tangent vector to the curve $\left.\left(x_{1}, x_{2}(t), \ldots, x_{k}(t)\right)\right)$ such that the derivative of the function $g$ along $\vec{V}$ is greater than 0 . Consequently $d g(A) \neq 0$.

We have just found $\varepsilon_{0}$ such that the neighborhood $U_{\varepsilon_{0}}^{0}\left(\Delta^{(0)}\right)$ does not contain critical points of the function $g$. Suppose we decrease $\varepsilon_{0}$. Note that we do not need to decrease the constructed neighborhood $U^{0}\left(\Delta^{(0)}\right)$ since the critical points of the function $g_{\varepsilon}$ with $\varepsilon<\varepsilon_{0}$ cannot appear inside it, because all the argumentation above works as well for any $\varepsilon<\varepsilon_{0}$.

Suppose we found $\varepsilon_{j-1}$ for a neighborhood $U^{k-j-1}\left(\Delta^{(k-j-1)}\right)$. Now chose $\delta$ by Lemma 2.2.4 with $\varepsilon=\varepsilon_{j-1}$ and put $\varepsilon_{j}=\frac{\delta}{j}$. Note that we need to consider not the entire $\Delta^{(k-j)}$, but only $\Delta^{(k-j)}-$ $U_{\varepsilon_{j-1}}^{k-j-1}\left(\Delta^{(k-j-1)}\right)$, i.e., we can assume that any point $A \in \Delta^{(k-j)}$ is of the form $\left(x_{1}, \ldots, x_{1}, x_{j+1}, \ldots, x_{k}\right)$, where $\left\|x_{1}-x_{j+1}\right\|>\varepsilon_{j-1}$ and $\left\|x_{1}-x_{k}\right\|>\varepsilon_{j-1}$. Arguing as above, we see that the whole neighborhood of $\Delta^{k-j}$ can be obtained if we fix $x_{1}, x_{j+1}, \ldots, x_{k}$ and put $x_{2}, \ldots, x_{j}$ on geodesics passing through $x_{1}$, thus $l\left(x_{1}, \ldots, x_{k}\right)=l(t)$. If $\left\|x_{i}-x_{i+1}\right\|<\frac{\delta}{j}$ for $i=1, \ldots, j-1$, then $\left\|x_{1}-x_{i}\right\|<\delta$ for 
$i=1, \ldots, j-1$ and by Lemma 2.2.4, it follows that $l^{\prime}(t)>0$. We have $g\left(x_{1}, \ldots, x_{k}\right)=g(t)$, and

$$
\begin{gathered}
g^{\prime}(t)=\underbrace{\left(\prod_{i=j}^{k} \varphi\left(\left\|x_{i}-x_{i+1}\right\|\right)\right)}_{\text {const }} \\
\cdot\left(\left(\prod_{i=1}^{j-1} \varphi\left(\left\|x_{i}-x_{i+1}\right\|\right)\right)^{\prime} l(t)+\left(\prod_{i=1}^{j-1} \varphi\left(\left\|x_{i}-x_{i+1}\right\|\right)\right) l^{\prime}(t)\right) .
\end{gathered}
$$

Since $l^{\prime}>0, l>0, \varphi>0, \varphi^{\prime} \geq 0$, it follows that $g^{\prime}(t)>0$ and $d g(A) \neq 0$ for any point $A \in U_{\varepsilon_{j}}^{j}\left(\Delta^{(k-j)}\right)$.

So we can pass from $\Delta^{(k-j-1)}$ to $\Delta^{(k-j)}$. Since $\Delta^{(k-2)}=\Delta$, this completes the proof.

Now let us introduce the following functions

$$
\begin{gathered}
f_{2}=\left\langle a, x_{1}-x_{2}\right\rangle: S^{n-1} \times M^{\times 2} \rightarrow \mathbb{R}, \\
f_{k}=\sum_{i \in \mathbb{Z}_{k}}\left\langle a_{i}, x_{i}-x_{i+1}\right\rangle:\left(S^{n-1}\right)^{\times k} \times M^{\times k} \rightarrow \mathbb{R}, k \geq 3,
\end{gathered}
$$

where $a, a_{i} \in S^{n-1}=\left\{u_{1}^{2}+\cdots+u_{n}^{2}=1\right\} \subset \mathbb{R}^{n}, x_{i} \in M$, and $i=i+k$ as usual. If $k \geq 3$, then there is an another expression for $f_{k}$ :

$$
f_{k}=\sum_{i \in \mathbb{Z}_{k}}\left\langle a_{i}-a_{i-1}, x_{i}\right\rangle .
$$

Now we are able to state and prove

Lemma 2.2.6. If $\left(x_{1}, \ldots, x_{k}\right)$ is a closed billiard trajectory, i.e., an isolated critical point of the function

$$
l_{k}=\sum_{i \in \mathbb{Z}_{k}}\left\|x_{i}-x_{i+1}\right\|: M^{\times k} \rightarrow \mathbb{R}
$$

then

$$
P_{0}=\left(a_{1}=\frac{x_{1}-x_{2}}{\left\|x_{1}-x_{2}\right\|}, \ldots, a_{k}=\frac{x_{k}-x_{1}}{\left\|x_{k}-x_{1}\right\|}, x_{1}, \ldots, x_{k}\right)
$$


is an isolated critical point of the function $f_{k}$. In accordance if $k=2$, then

$$
P_{0}=\left(a=\frac{x_{1}-x_{2}}{\left\|x_{1}-x_{2}\right\|}, x_{1}, x_{2}\right)
$$

is the corresponding critical point of the function $f_{2}$.

Proof. Let us start from investigating isolated critical points of the function $f_{k}$. Consider such a critical point $P_{0}=\left(a_{1}, \ldots, a_{k}, x_{1}, \ldots, x_{k}\right)$, where $x_{i} \neq x_{i+1}$ for every $i \in \mathbb{Z}_{k}$. Then $\frac{\partial f_{k}}{\partial a_{i}}\left(P_{0}\right)=0$ for all $i$ and $\frac{\partial f_{k}}{\partial x_{i}}\left(P_{0}\right)=0$ for all $i$. The first condition gives us

$$
x_{i}-x_{i+1} \perp T_{a_{i}} S^{n-1}
$$

that is $a_{i} \| x_{i}-x_{i+1}$, while the second one means that

$$
a_{i-1}-a_{i} \perp T_{x_{i}} M \text {. }
$$

Now return to critical points of $l_{k}$. Let $\left(x_{1}, \ldots, x_{k}\right)$ be one of them. By the definition of a closed billiard trajectory, we have

$$
\frac{x_{i}-x_{i+1}}{\left\|x_{i}-x_{i+1}\right\|}-\frac{x_{i-1}-x_{i}}{\left\|x_{i-1}-x_{i}\right\|} \perp T_{x_{i}} M
$$

Putting $a_{i}=\frac{x_{i}-x_{i+1}}{\left\|x_{i}-x_{i+1}\right\|}$ completes the proof.

Remark 2.2.1. If $k>2$, then the points coming from the closed billiard trajectories are not the only critical points of the function $f_{k}$.

Remark 2.2.2. In fact, to each closed billiard trajectory $\left(x_{1}, \ldots, x_{k}\right)$ corresponds $4 k$ critical points of the function $f_{k}$ since there is a symmetry given by the action of the group $\mathbb{Z}_{2} \oplus D_{k}$. Here $\mathbb{Z}_{2}$ acts by the reflection

$$
\left(a_{1}, \ldots, a_{k}, x_{1}, \ldots, x_{k}\right) \rightarrow\left(-a_{1}, \ldots,-a_{k}, x_{1}, \ldots, x_{k}\right),
$$

while $D_{k}$ acts by permuting simultaneously $a_{1}, \ldots, a_{k}$ and $x_{1}, \ldots, x_{k}$.

Lemma 2.2.7. Suppose $P_{0}=\left(a_{1}, \ldots, a_{k}, x_{1}, \ldots, x_{k}\right)$ is a critical point of the function $f_{k}, k>2$ and some of the points $x_{i}$ coincide. Namely let $\beta_{1}, \ldots, \beta_{k^{\prime}}$ be integers such that

1. $\beta_{1}, \ldots, \beta_{k^{\prime}} \geq 1$ 
2. $\beta_{1}+\cdots+\beta_{k^{\prime}}=k$,

3. $1<k^{\prime}<k$.

By $\alpha_{i}$ denote the sum $\alpha_{i}=\beta_{1}+\cdots+\beta_{i}$. Then let $x_{i}$ s satisfy:

$$
\begin{aligned}
& x_{1}=\cdots=x_{\alpha_{1}} \neq \\
& x_{\alpha_{1}+1}=\cdots=x_{\alpha_{2}} \neq \\
& \vdots \\
& x_{\alpha_{k^{\prime}-1}+1}=\cdots=x_{k} \neq x_{1}
\end{aligned}
$$

Then the following conditions hold:

1. the point $P_{0}^{\prime}=\left(a_{1}, a_{\alpha_{1}+1}, \ldots, a_{\alpha_{k^{\prime}-1}+1}, x_{\alpha_{1}}, \ldots, x_{\alpha_{k^{\prime}}}\right)$ is a critical point of the function $f_{k^{\prime}}$.

2. the point $P_{0}$ belongs to a critical manifold $M_{0}$ given by the following conditions

$$
\begin{aligned}
& a_{2}-a_{1}, \ldots, a_{\alpha_{1}}-a_{\alpha_{1}-1}, a_{\alpha_{1}+1}-a_{\alpha_{1}} \perp T_{x_{\alpha_{1}}} M \\
& a_{\alpha_{1}+2}-a_{\alpha_{1}+1}, \ldots, a_{\alpha_{2}}-a_{\alpha_{2}-1}, a_{\alpha_{2}+1}-a_{\alpha_{2}} \perp T_{x_{\alpha_{2}}} M, \\
& \vdots \\
& a_{\alpha_{k^{\prime}-1}+2}-a_{\alpha_{k^{\prime}-1}+1}, \ldots, a_{\alpha_{k^{\prime}}}-a_{\alpha_{k^{\prime}-1}}, a_{1}-a_{k} \perp T_{x_{k}} M,
\end{aligned}
$$

Proof. Without loss of generality, consider the simplest case $x_{1}=x_{2}$ and $x_{i} \neq x_{i+1}$ for $i \neq 1$. As above, we obviously have

$$
a_{i} \| x_{i}-x_{i+1}, \quad i \neq 1
$$

and

$$
a_{i}-a_{i+1} \perp T_{x_{i}} M, \quad i \in \mathbb{Z}_{k}
$$

At the vertex $x_{1}=x_{2}$ we have $a_{k}-a_{1} \perp T_{x_{1}} M$ and $a_{1}-a_{2} \perp T_{x_{1}} M$. Summing these two conditions, we get $a_{k}-a_{2} \perp T_{x_{1}} M$ and the possible positions for $a_{1}$ form a sphere of dimension $n-m-1$. If $n=m+1$, then this sphere is just a couple of points, but anyway for further considerations we need only the case $n \geq m+2$, that's why we do not pay much attention to $n=m+1$. 
If $k^{\prime}<k-1$, then exactly the same argumentation proves the lemma as well. In additional let us notice that critical manifolds are products of spheres. Indeed, consider, for example, the set of conditions

$$
\begin{aligned}
& a_{2}-a_{1} \perp T_{x_{\alpha_{1}}} M, \\
& \vdots \\
& a_{\alpha_{1}}-a_{\alpha_{1}-1} \perp T_{x_{\alpha_{1}}} M, \\
& a_{\alpha_{1}+1}-a_{\alpha_{1}} \perp T_{x_{\alpha_{1}}} M .
\end{aligned}
$$

Here $a_{1}$ and $a_{\alpha_{1}+1}$ are fixed, while all other $a_{i}$ can be chosen freely to satisfy these conditions. It gives us a product $\left(S^{n-m-1}\right)^{\times\left(\alpha_{1}-1\right)}$.

Remark 2.2.3. There is also a critical manifold $M^{(0)}$ given by

$$
\begin{aligned}
& x_{1}=\cdots=x_{k}=x \in M, \\
& a_{i}-a_{j} \perp T_{x} M, i \neq j .
\end{aligned}
$$

It is a bundle over $M$ with a fiber defined by the 2 nd condition.

Lemma 2.2.8. Suppose $\left(x_{1}, \ldots, x_{k}\right)$ is an isolated non-degenerate critical point of the function $l_{k}$ and its Morse index is $\mu$. Then the corresponding critical point

$$
P_{0}=\left(a_{1}, \ldots, a_{k}, x_{1}, \ldots, x_{k}\right), a_{i}=\frac{x_{i}-x_{i+1}}{\left\|x_{i}-x_{i+1}\right\|},
$$

of the function $f_{k}$ is also non-degenerate, its Morse index is $\mu+k(n-1)$ for $k>2$ and $\mu+n-1$ for $k=2$.

Proof. Suppose a point $P=\left(b_{1}, \ldots, b_{k}, y_{1} \ldots, y_{k}\right)$ belongs to a neighborhood of the critical point $P_{0}$ being considered. Let us introduce coordinates in a small neighborhood of $P_{0}$. Suppose

$$
y_{i}=y_{i}\left(t_{i}\right),
$$

where $t_{i} \in \mathbb{R}^{m}$ is some parametrization for $y_{i}$.

$$
b_{i}=b_{i}\left(s_{i}\right),
$$

where $s_{i}$ is a parametrization for $b_{i}$ defined as follows. Let $A_{i}$ be some orthogonal operator $\mathbb{R}^{n-1} \rightarrow\left(y_{i}-y_{i+1}\right)^{\perp}, s_{i} \in \mathbb{R}^{n-1}$ is our parameter. Put

$$
b_{i}=\frac{\left\|s_{i}\right\| A_{i} s_{i}+\frac{y_{i}-y_{i+1}}{\left\|y_{i}-y_{i+1}\right\|}}{\|\| s_{i}\left\|A_{i} s_{i}+\frac{y_{i}-y_{i+1}}{\left\|y_{i}-y_{i+1}\right\|}\right\|}=\frac{\left\|s_{i}\right\| A_{i} s_{i}+\frac{y_{i}-y_{i+1}}{\left\|y_{i}-y_{i+1}\right\|}}{\sqrt{1+\left\|s_{i}\right\|^{2}}}
$$


Evidently, $\left\|b_{i}\right\|=1$ and $b(0)=\frac{y_{i}-y_{i+1}}{\left\|y_{i}-y_{i+1}\right\|}$. Now since $A_{i} s_{i} \perp y_{i}-y_{i+1}$, we have

$$
\left\langle b_{i}, y_{i}-y_{i+1}\right\rangle=\frac{\left\|y_{i}-y_{i+1}\right\|}{\sqrt{1+\left\|s_{i}\right\|^{2}}} .
$$

Thus the following equality holds

$$
\begin{aligned}
f_{k}(P) & =\sum_{i \in \mathbb{Z}_{k}}\left\langle b_{i}, y_{i}-y_{i+1}\right\rangle=\sum_{i \in \mathbb{Z}_{k}} \frac{\left\|y_{i}-y_{i+1}\right\|}{\sqrt{1+\left\|s_{i}\right\|^{2}}} \\
& =l_{k}\left(y_{1}, \ldots, y_{k}\right)-\frac{1}{2} \sum\left\|s_{i}\right\|^{2}+\ldots
\end{aligned}
$$

This concludes the proof. $\square$

Remark 2.2.4. Suppose $x_{i}=x_{i+1}$ for some $i$. As we have showed above, in this case there are some critical manifolds corresponding to critical points of the function $f_{k^{\prime}}, k^{\prime}<k$. If $\left(x_{1}, \ldots, x_{k^{\prime}}\right)$ is a nondegenerate critical point of a function $l_{k^{\prime}}$, then the corresponding critical manifold is also non-degenerate. The critical manifold $M^{(0)}$ defined by $x_{1}=x_{2}=\cdots=x_{k}$ is non-degenerate as well.

Lemma 2.2.9. Suppose $F: M \times[0,1] \rightarrow \mathbb{R}^{n}$ is a smooth homotopy such that for every $t$ the embedding $F_{t}: M \rightarrow \mathbb{R}^{n}$ is generic, $k \geq 2$ an integer. Then the homotopy $F$ keeps the number of closed billiard trajectories with $k$ vertices.

Proof. Consider the homotopy $F: M \times[0,1] \rightarrow \mathbb{R}^{n}$. Denote $f_{k^{-}}$and $l_{k}$-functions corresponding to an embedding $F_{t}$ by $f_{k t}$ and $l_{k t}$.

From the previous statements we know that the whole picture is as follows. Closed billiard trajectories (that are non-degenerate isolated critical points of the function $l_{k}$ ) correspond to non-degenerate critical points of the function $f_{k}$ that is a smooth function defined on the smooth manifold

$$
\left(S^{n-1}\right)^{\times k} \times M^{\times k}
$$

We suppose every embedding $F_{t}: M \rightarrow \mathbb{R}^{n}$ to be generic, thus $f_{k t}$ has an amount of isolated critical points and several non-degenerate critical manifolds corresponding to isolated critical points of the functions $f_{k^{\prime} t}$ with $k^{\prime}<k$. 
Thus when the embedding $F_{0}: M \rightarrow \mathbb{R}^{n}$ is being deformed, isolated critical points of the function $f_{k}$ could disappear and be born only from non-degenerate critical manifolds that is impossible. Indeed, suppose an isolated critical point is born at $t=t_{0}$. We mean that there exists $M_{t}$ - a non-degenerate critical manifold of the function $f_{k t}$ for $\left|t-t_{0}\right|$ small enough and for $t>t_{0}$ there exists an isolated critical point $P_{t}$ such that $\lim _{t \rightarrow t_{0}+0} P_{t}=P_{0} \in M_{t_{0}}$. By Morse-Bott theory, there are coordinates $t, X^{1}, \ldots, X^{N}$ in a neighborhood $U \subset\left(S^{n-1}\right)^{\times k} \times M^{\times k} \times\left[t_{0}-\varepsilon, t_{0}+\varepsilon\right]$ of the point $P_{t_{0}}$ such that $M_{t}$ is given by $X^{1}=\cdots=X^{r}=0$ and

$$
f_{k t}=C(t)-\left(X^{r+1}\right)^{2}-\cdots-\left(X^{r+s}\right)^{2}+\left(X^{r+s+1}\right)^{2}+\cdots+\left(X^{N}\right)^{2} .
$$

We see that in the neighborhood $U$ there are no other isolated critical points of the function $f_{k t}$. This contradiction completes the proof.

Corollary 2.2.10. Generic embeddings form an open set in the space of all smooth embeddings $M \rightarrow \mathbb{R}^{n}$.

\subsection{Generic embeddings}

Finally, let us prove that generic embeddings are really generic: they form an open dense set in the space of all embeddings. We have shown above that they form an open set, so what remains is the following

Theorem 2.3.1. Let a smooth closed m-dimensional manifold $M$, an integer $k \geq 2$, and an integer $n>m$ be fixed. If there exists some embedding e $: M \rightarrow \mathbb{R}^{n}$, then $k$-generic embeddings form a dense set in the space of all smooth embeddings $M \rightarrow \mathbb{R}^{n}$.

Proof. Let $e: M \rightarrow \mathbb{R}^{n}$ be some embedding. We need to show that $e$ can be perturbed slightly to become $k$-generic. Let us prove it by induction. The inductive base $k=2$ is proved by Takens and White in [4].

Suppose now $e$ to be $(k-1)$-generic. Recall that $\Delta \subset M^{\times k}$ is the diagonal consisting of all the points $\left\{\left(x_{1}, \ldots, x_{k}\right)\right\}$ such that $x_{i}=x_{i+1}$ for some $i$ or $x_{1}=x_{k}$. Notice that there exists $\varepsilon>0$ such that the neighborhood

$$
U_{\varepsilon}(\Delta)=\bigcup_{i \in \mathbb{Z}_{k}}\left\{\left(x_{1}, \ldots, x_{k}\right):\left\|x_{i}-x_{i+1}\right\|<\varepsilon\right\}
$$


does not contain critical points of the function $l_{k}$. Indeed, suppose the opposite: a sequence of critical points $P_{1}, P_{2}, P_{3}, \ldots$ converges to some point $P \in \Delta$. Let us denote corresponding critical points of the function $f_{k}$ by $\tilde{P}_{1}, \tilde{P}_{2}, \tilde{P}_{3}, \ldots, \tilde{P} \in\left(S^{n-1}\right)^{\times k} \times M^{\times k}$. By lemma 2.2.7, $\tilde{P}$ corresponds to some critical point $\tilde{P}^{\prime} \in\left(S^{n-1}\right)^{\times k^{\prime}} \times M^{\times k^{\prime}}$ of some function $f_{k^{\prime}}$ with $k^{\prime}<k$. By the inductive assumption, $\tilde{P}^{\prime}$ is a nondegenerate critical point. Therefore $\tilde{P}$ belongs to some non-degenerate critical manifold and such a sequence $\tilde{P}_{1}, \tilde{P}_{2}, \tilde{P}_{3}, \ldots$ cannot exist.

Put $X=M^{\times k} \backslash U_{\varepsilon}(\Delta)$. Let $\tilde{d} l: X \rightarrow T^{*} X$ be a mapping defined by the formula

$$
\tilde{d l}(P)=d l_{k}(P) .
$$

By $X_{0} \subset T^{*} X$ denote a zero section of the cotangent bundle $T^{*} X$. Thus our goal is to perturb the embedding $e$ such that the mapping $\tilde{d} l$ becomes transversal to $X_{0}$.

We do it introducing a manifold of parameters $A$ and a mapping $E: A \times M \rightarrow \mathbb{R}^{n}$ such that each $E_{a}(x)=E(a, x): M \rightarrow \mathbb{R}^{n}$ is an embedding, $E_{0}=e$. Let

$$
\begin{gathered}
l_{a}\left(x_{1}, \ldots, x_{k}\right)=\left\|E_{a}\left(x_{1}\right)-E_{a}\left(x_{2}\right)\right\|+\left\|E_{a}\left(x_{2}\right)-E_{a}\left(x_{3}\right)\right\|+\ldots \\
\left\|E_{a}\left(x_{k-1}\right)-E_{a}\left(x_{k}\right)\right\|+\left\|E_{a}\left(x_{k}\right)-E_{a}\left(x_{1}\right)\right\|
\end{gathered}
$$

be a corresponding length function. By $L: A \times X \rightarrow T^{*} X$ denote a mapping defined by the formula $L_{a}(P)=L(a, P)=d l_{a}(P)$, where $d l_{a}$ is a differential of the function $l_{a}$ with $a$ fixed. We construct $A$ and $E$ such that $L$ is transversal to $X_{0}$. Then by a well known theorem, the set of all $a \in A$ such that $L_{a}$ is transversal to $X_{0}$ is dense in $A$. Thus the perturbation we need is $E_{a}$ for some $a$ close to 0 such that $L_{a}$ is transversal to $X_{0}$. The only thing remained to complete the proof is to construct $A$ and $E$.

Let $M=U_{1} \cup \cdots \cup U_{N}$ be a finite open covering, each $U_{i}$ is a coordinate neighborhood of diameter not exceeding $\varepsilon$. Let $V_{i} \subset U_{i}$ be open sets such that $\overline{V_{i}} \subset U_{i}$ and $M=V_{1} \cup \cdots \cup V_{N}$. Let $\varphi_{i}$ be a function $M \rightarrow \mathbb{R}$ satisfying

1. $0 \leq \varphi_{i} \leq 1$,

2. $\left.\varphi\right|_{V_{i}} \equiv 1$,

3. $\left.\varphi\right|_{M \backslash U_{i}} \equiv 0$. 
Let $a_{1}, \ldots, a_{N} \in \mathbb{R}^{n}$ be some vectors. We perturb $e$ as follows

$$
E(a, x)=e(x)+\sum_{i=1}^{N} \varphi_{i} a_{i}
$$

If all $\left\|a_{i}\right\|$ are small enough, then $E_{a}$ is an embedding. Let $A$ be a small neighborhood of 0 in the vector space $\mathbb{R}^{N n}$.

Suppose now $P=\left(x_{1}, \ldots, x_{k}\right) \in X$ is a critical point of $l_{a}$ for some $a \in A$. Then each $x_{i} \in V_{\alpha_{i}}$ such that for any $i \in \mathbb{Z}_{k} \alpha_{i} \neq \alpha_{i+1}$ (else $P$ would belong to $\left.U_{\varepsilon}(\Delta)\right)$. Let us compute the derivatives of the function $l_{a}(P)=l_{a}\left(x_{1}, \ldots, x_{k}\right)$. First,

$$
\frac{\partial l_{a}}{\partial x_{i}}(P)=\sum_{i \in \mathbb{Z}_{k}} \frac{E_{a}\left(x_{i}\right)-E_{a}\left(x_{i-1}\right)}{\left\|E_{a}\left(x_{i}\right)-E_{a}\left(x_{i-1}\right)\right\|}+\frac{E_{a}\left(x_{i}\right)-E_{a}\left(x_{i+1}\right)}{\left\|E_{a}\left(x_{i}\right)-E_{a}\left(x_{i+1}\right)\right\|} .
$$

Second,

$$
\frac{\partial^{2} l_{a}}{\partial x_{i} \partial a_{\alpha_{i}}}(P)=\frac{a_{\alpha_{i}}}{\left\|E_{a}\left(x_{i}\right)-E_{a}\left(x_{i-1}\right)\right\|}+\frac{a_{\alpha_{i}}}{\left\|E_{a}\left(x_{i}\right)-E_{a}\left(x_{i+1}\right)\right\|} .
$$

Since $a_{\alpha_{i}}$ can be chosen freely, we obtain that $d L\left(T_{P} X\right)$ gives the whole $T_{(P, 0)}\left(T^{*} X\right) / T_{P}\left(X_{0}\right)$. This completes the proof. 
Chapter 3

\section{Homology of symmetric products}




\subsection{A. Dold's theory}

In the rest of this work we will give some estimates for the sum of Betti numbers. Our approach follows A. Dold, who proved an interesting result about the homology of symmetric products of a topological space using his machinery of face degeneracy modules ("FD-modules"). Here we shortly set out his theory.

In this section by the word module we mean a $\mathbb{Z}_{2}$-module that is a vector space over the field consisting of two elements. In fact, all the results below hold for a much wider class of modules, but we apply all this staff only to homology groups with $\mathbb{Z}_{2}$-coefficients.

Definition 3.1.1. An FD-module $K$ is a sequence of modules $K_{q}$, $q=0,1, \ldots$ and module morphisms $\partial_{q}^{i}: K_{q} \rightarrow K_{q-1}, s_{q}^{i}: K_{q} \rightarrow K_{q+1}$, $i=0,1, \ldots$ The morphisms $\partial_{q}^{i}$ are called face operators, $s_{q}^{i}$ are called degeneracy operators. The face- and degeneracy- operators satisfy the following axioms:

1. $\partial_{q}^{i}=0, s_{q}^{i}=0$ if $i>q$.

2. $\partial_{q-1}^{i} \partial_{q}^{j}=\partial_{q-1}^{j-1} \partial_{q}^{i}$ if $i<j$.

3. $s_{q+1}^{i} s_{q}^{j}=s_{q+1}^{j+1} s_{q}^{i}$ if $i \leq j$.

4. $\partial_{q+1}^{i} s_{q}^{j}=s_{q-1}^{j-1} \partial_{q}^{i}$ if $i<j$.

5. $\partial_{q+1}^{i} s_{q}^{i}=\partial_{q+1}^{i+1} s_{q}^{i}=\mathrm{I} d$ if $i \leq q$.

6. $\partial_{q+1}^{i} s_{q}^{j}=s_{q-1}^{j} \partial_{q}^{i-1}$ if $i>j+1$.

Now let us recall the well-known

Definition 3.1.2. A chain module $C$ is a sequence of modules $C_{q}$, $q=0,1, \ldots$ and module morphisms $\partial_{q}: C_{q} \rightarrow C_{q-1}, q=1,2, \ldots$ such that $\partial_{q} \partial_{q+1}=0$ for all $q$. The morphisms $\partial_{q}$ are called boundary operators. 
Definition 3.1.3. Given an FD-module $K$, put

$$
R(K)_{q}=\bigcap_{i<q} \operatorname{ker}\left(\partial_{i}^{q}: K_{q} \rightarrow K_{q-1}\right), \quad q=0,1, \ldots
$$

Clearly, $\partial_{q}^{q}\left(R(K)_{q}\right) \subset R(K)_{q-1}$ and $\left.\partial_{q-1}^{q-1} \partial_{q}^{q}\right|_{R(K)_{q}}=0$. So $R$ is a functor from FD-modules to chain modules. Homology groups are

$$
H_{q}(K)=H_{q}(R(K))=\operatorname{ker} \partial_{q}^{q} / \operatorname{im} \partial_{q+1}^{q+1} .
$$

Another way to define homology groups is as follows. The boundary operator is $\partial_{q}=\partial_{q}^{0}-\partial_{q}^{1}+\partial_{q}^{2}-\ldots$, the $q$ th homology group is $H_{q}=$ $\operatorname{ker} \partial_{q} / \mathrm{im} \partial_{q+1}$. Denote this functor by $R^{\prime}$.

Proposition 3.1.1. (A. Dold, [11])

1. There exists a functor $R^{-1}$ from chain modules to FD-modules such that $R R^{-1}$ and $R^{-1} R$ are naturally equivalent to the respective identity functors.

2. Let $K$ be an FD-module. Chain modules $R(K)$ and $R^{\prime}(K)$ are homotopically equivalent $t^{1}$ and therefore they have isomorphic homology groups.

3. The functor $R$ moves homotopically equivalent FD-modules to homotopically equivalent chain modules.

A morphism of FD-modules (or FD-morphism) $i: K \rightarrow K^{\prime}$ is a set of linear mappings $i_{q}: K_{q} \rightarrow K_{q}^{\prime}$ such that $i_{q-1} \partial^{i}=\partial^{i} i_{q}$ and $i_{q+1} s^{i}=s^{i} i_{q}$. A morphism of FD-modules induces a homomorphism of homology groups $i_{*}$.

Example 3.1.1. Let $\Upsilon_{q}$ be the standard $q$-dimensional simplex:

$$
\Upsilon_{q}=\left\{\left(x_{0}, \ldots, x_{q}\right) \in \mathbb{R}^{q+1}: x_{i} \geq 0, \sum x_{i}=1\right\} .
$$

Introduce the mappings

$$
\begin{gathered}
\varepsilon_{q}^{i}: \Upsilon_{q} \rightarrow \Upsilon_{q+1}, \quad \varepsilon_{q}^{i}\left(x_{0}, \ldots, x_{q}\right)=\left(x_{0}, \ldots, x_{i-1}, 0, x_{i}, \ldots, x_{q}\right), \\
\eta_{q}^{i}: \Upsilon_{q} \rightarrow \Upsilon_{q-1}, \quad \eta_{q}^{i}\left(x_{0}, \ldots, x_{q}\right)=\left(x_{0}, \ldots, x_{i-1}+x_{i}, x_{i+1}, \ldots, x_{q}\right) .
\end{gathered}
$$

\footnotetext{
${ }^{1}$ The notion of homotopic equivalence is defined later
} 
Suppose $X$ is a topological space, $K_{q}$ is the group of its singular $q$ chains. The elements of $K_{q}$ are finite sums $\sum a_{k} f_{k}$, where $f_{k}: \Upsilon_{q} \rightarrow X$ are continuous maps. Now introduce face- and degeneracy-operators:

$$
\begin{aligned}
& \partial_{q}^{i}(f)=f \circ \varepsilon_{q-1}^{i}, \\
& s_{q}^{i}(f)=f \circ \eta_{q+1}^{i} .
\end{aligned}
$$

We have constructed the FD-module $K(X)$ of a topological space $X$.

Let $x_{0}$ be a distinguished point of the space $X$. All simplices $\left\{f: \Upsilon_{q} \rightarrow x_{0}\right\}$ form an FD-submodule $p t(X) \subset K(X)$. The quotient $K(X) / p t(X)$ is called the reduced $F D$-module of a topological space $X$, its homology $\tilde{H}_{*}(X)$ is the reduced homology of $X$. It is well known that $\tilde{H}_{q}(X) \cong H_{q}(X)$ if $q>0, \operatorname{dim} H_{0}(X)=\operatorname{dim} \tilde{H}_{0}(X)+1$.

The continuous map $f: X \rightarrow Y$ induces an FD-morphism $f_{\#}$ : $K(X) \rightarrow K(Y)$. The map $f:\left(X, x_{0}\right) \rightarrow\left(Y, y_{0}\right)$ of pointed spaces induces a morphism of reduced FD-modules.

Now we define a direct product of FD-modules.

Definition 3.1.4. Let $K^{\prime}$ and $K^{\prime \prime}$ be FD-modules. Put

$$
\begin{gathered}
\left(K^{\prime} \times K^{\prime \prime}\right)_{q}=K_{q}^{\prime} \otimes K_{q}^{\prime \prime}, \\
\partial_{q}^{i}\left(a_{q}^{\prime} \otimes a_{q}^{\prime \prime}\right)=\partial_{q}^{i}\left(a_{q}^{\prime}\right) \otimes \partial_{q}^{i}\left(a_{q}^{\prime \prime}\right), s_{q}^{i}\left(a_{q}^{\prime} \otimes a_{q}^{\prime \prime}\right)=s_{q}^{i}\left(a_{q}^{\prime}\right) \otimes s_{q}^{i}\left(a_{q}^{\prime \prime}\right) .
\end{gathered}
$$

Let $X^{\prime}$ and $X^{\prime \prime}$ be topological spaces. Clearly, $K\left(X^{\prime} \times X^{\prime \prime}\right) \cong$ $K\left(X^{\prime}\right) \times K\left(X^{\prime \prime}\right)$. Indeed, let $\alpha: X^{\prime} \times X^{\prime \prime} \rightarrow X^{\prime}$ and $\beta: X^{\prime} \times X^{\prime \prime} \rightarrow X^{\prime \prime}$ be the projections of the Cartesian product onto each factor. Then $\alpha_{\#} \times \beta_{\#}: K\left(X^{\prime} \times X^{\prime \prime}\right) \rightarrow K\left(X^{\prime}\right) \times K\left(X^{\prime \prime}\right)$ is an isomorphism of FDmodules.

Now define an FD-module homotopy. First we construct an FDmodule $K(n)$ that is usually called the FD-module of the standard $n$-simplex. The group $K(n)_{q}$ is free generated by all sets of integers $\left(w_{0}, w_{1}, \ldots, w_{q}\right)$ such that $0 \leq w_{0} \leq \cdots \leq w_{q} \leq n$. Face- and degeneracy- operators are given by the formulas

$$
\begin{gathered}
\partial_{q}^{i}\left(w_{0}, \ldots, w_{q}\right)=\left(w_{0}, \ldots, w_{i-1}, w_{i+1}, \ldots, w_{q}\right), \\
s_{q}^{i}\left(w_{0}, \ldots, w_{q}\right)=\left(w_{0}, \ldots, w_{i}, w_{i}, \ldots, w_{q}\right) .
\end{gathered}
$$

Let $e_{i}=(i) \in K(n)_{0}$ be an $i$ th vertex. 
Definition 3.1.5. Suppose $K^{\prime}$ and $K^{\prime \prime}$ are FD-modules, $F^{0}, F^{1}$ : $K^{\prime} \rightarrow K^{\prime \prime}$ are FD-morphisms. An FD-homotopy is an FD-morphism $\Theta: K(1) \times K^{\prime} \rightarrow K^{\prime \prime}$ such that

$$
\Theta\left(\left(s_{0}^{0}\right)^{q} e_{i} \otimes a_{q}\right)=F^{i}\left(a_{q}\right), a_{q} \in K_{q}^{\prime}, i=0,1 .
$$

If there exists such homotopy $\Theta$, then FD-morphisms $F^{0}$ and $F^{1}$ are called homotopic, $F^{0} \sim F^{1}$. If there exist FD-morphisms $F^{+}: K^{\prime} \rightarrow$ $K^{\prime \prime}$ and $F^{-}: K^{\prime \prime} \rightarrow K^{\prime}$ such that $F^{+} F^{-} \sim \mathrm{I} d$ and $F^{-} F^{+} \sim \mathrm{I} d$, then FD-modules $K^{\prime}$ and $K^{\prime \prime}$ are called homotopically equivalent.

A. Dold has proved the following fact in [11]:

Theorem 3.1.2. Two FD-modules are homotopically equivalent if and only if they have isomorphic homology groups.

Let $T$ be a functor on the category of FD-modules.

Definition 3.1.6. The functor $T$ preserves homotopy if $T\left(F^{0}\right) \sim$ $T\left(F^{1}\right)$ for each pair $F^{0}, F^{1}$ of homotopic FD-morphisms.

Theorem 3.1.3. (A. Dold, [11]) Suppose the functor T preserves homotopy. Then

$$
H_{*}\left(K^{\prime}\right) \cong H_{*}\left(K^{\prime \prime}\right) \text { implies } H_{*}\left(T\left(K^{\prime}\right)\right) \cong H_{*}\left(T\left(K^{\prime \prime}\right)\right),
$$

for each pair of FD-modules $K^{\prime}, K^{\prime \prime}$.

Let $t$ be a functor on the category of $\mathbb{Z}_{2}$-modules. Suppose $K$ is an FD-module. Put

$$
T(K)_{q}=t\left(K_{q}\right), \quad F_{q}^{i}=t\left(\partial_{q}^{i}\right), \quad D_{q}^{i}=t\left(s_{q}^{i}\right) .
$$

It is easy to check that $T(K)$ is an FD-module with face-operators $F_{q}^{i}$ and degeneracy-operators $D_{q}^{i}$. We say that the functor $T$ is a prolongation of the functor $t$.

The $p$ th direct power $K^{\times p}$ of an FD-module $K$ is the simplest example of a prolongation.

Theorem 3.1.4. (A. Dold, [11]) Suppose the functor $T$ is a prolongation of the functor $t$. Then $T$ preserves homotopy. 


\subsection{Lemma on symmetric products}

Now we are able to prove the central statement in this chapter.

Definition 3.2.1. A subset $X$ of a Euclidean space $\mathbb{R}^{N}$ is said to be a polytope if $X=\bigcup L_{i}^{q}$ such that

- $L_{i}^{q}$ is affine equivalent to a standard $q$-simplex $\Upsilon_{q}$ that is given by $x_{0}+x_{1}+\cdots+x_{q}=0$, all $x_{i} \geq 0$.

- any intersection $L_{i}^{q} \cap L_{i^{\prime}}^{q^{\prime}}$ is either empty set or a union of $L_{i^{\prime \prime}}^{q^{\prime \prime}}$ of less dimension $q^{\prime \prime}<q, q^{\prime}$.

Definition 3.2.2. A subset $X$ of a Euclidean space $\mathbb{R}^{N}$ is said to be a (convex) polyhedron if

- $X$ is a compact set.

- $X$ is given by a finite number of linear equations and inequalities.

Let us recall the following notation. Suppose $k>1$ is an integer and $X$ is a topological space. Put

$$
\Delta_{X}=\bigcup_{i \in \mathbb{Z}_{k}}\left\{\left(x_{1}, \ldots, x_{k}\right): x_{i}=x_{i+1}\right\} \subset X^{\times k} .
$$

The dihedral group $D_{k}$ acts on $M^{\times k}$ by permutations of factors. We denote the quotient space $\Delta_{X} / D_{k}$ also by $\Delta_{X}$.

Lemma 3.2.1. Let $M_{1}$ and $M_{2}$ be polytopes, $k$ a prime integer. Then

$$
H_{*}\left(M_{1} ; \mathbb{Z}_{2}\right) \cong H_{*}\left(M_{2} ; \mathbb{Z}_{2}\right)
$$

implies

$$
H_{*}\left(M_{1}^{\times k} / D_{k}, \Delta_{M_{1}} ; \mathbb{Z}_{2}\right) \cong H_{*}\left(M_{2}^{\times k} / D_{k}, \Delta_{M_{2}} ; \mathbb{Z}_{2}\right) .
$$

Proof. Suppose $M$ is a triangulated subset of a Euclidean space, $P \in M$ is a distinguished point. Thus $(P, \ldots, P)$ is a distinguished point in the Cartesian degree $M^{\times k}$. Let $X$ be quotient space $M^{\times k} / D_{k}$ with the diagonal $\Delta_{M}$ contracted to a point. 
Let us introduce the following notations:

$$
\begin{gathered}
K=K\left(M^{\times k}\right) / p t\left(M^{\times k}\right) \cong(K(M) / p t(M))^{\times k},{ }^{2} \\
K^{\prime}=K(X) / p t(X)
\end{gathered}
$$

The projection $\pi: M^{\times k} \rightarrow X$ induces an FD-morphism $\pi_{\#}: K \rightarrow$ $K^{\prime}$.

By $K_{\text {sym }}$ denote the FD-submodule of $K$ generated by all chains of the form $c_{1} \otimes c_{2} \otimes \cdots \otimes c_{k}+c_{2} \otimes \cdots \otimes c_{k} \otimes c_{1}, c_{1} \otimes c_{2} \otimes \cdots \otimes c_{k}+$ $c_{k} \otimes \cdots \otimes c_{2} \otimes c_{1}$, and $c \otimes c \otimes c_{3} \otimes \cdots \otimes c_{k}$. Let $u: K_{\text {sym }} \rightarrow K$ be the inclusion, $\varphi: K \rightarrow K / K_{\text {sym }}$ be the quotient FD-morphism.

It follows from A. Dold's theorem that the homology $H_{*}\left(K / K_{\text {sym }}\right)$ can be computed if we know only the homology $H_{*}(M)$. Indeed, let $\kappa$ be a $\mathbb{Z}_{2}$-module. By $t(\kappa)$ denote the quotient of $\kappa \otimes \cdots \otimes \kappa$ by the relations $c_{1} \otimes c_{2} \otimes \cdots \otimes c_{k}+c_{2} \otimes \cdots \otimes c_{k} \otimes c_{1}=0, c_{1} \otimes c_{2} \otimes \cdots \otimes c_{k}+c_{k} \otimes \cdots \otimes c_{2} \otimes c_{1}=0$, and $c \otimes c \otimes c_{3} \otimes \cdots \otimes c_{k}=0$. Let $T$ be the prolongation of the functor $t$. Now we see that the correspondence $K(M) \rightarrow K / K_{\text {sym }}$ is the composition of the factorization $K(M) \rightarrow K(M) / p t(M)$ and the functor $T$.

Let us prove that $H_{*}\left(K / K_{\text {sym }}\right) \cong H_{*}\left(K^{\prime}\right)$, this will complete the proof of the lemma.

First we show that $\alpha=\pi_{\#} \circ \varphi^{-1}$ is an FD-morphism. We need to check that $u\left(K_{\text {sym }}\right) \subset \operatorname{ker} \pi_{\#}$.

Let $c=f^{1} \otimes \cdots \otimes f^{k}, c^{\prime}=f^{2} \otimes \cdots \otimes f^{k} \otimes f^{1}$ be two elements of $K_{q}$. Here $f^{1}, \ldots, f^{k}: \Upsilon_{q} \rightarrow M$ are continuous maps of the standard $q$-simplex to the space $M$. Evidently, $\pi_{\#}(c)=\pi_{\#}\left(c^{\prime}\right)$, hence $c+c^{\prime} \in$ $\operatorname{ker} \pi_{\#}$. Now suppose $c=\left(\sum f_{i_{1}}^{1}\right) \otimes \cdots \otimes\left(\sum f_{i_{k}}^{k}\right), c^{\prime}=\left(\sum f_{i_{2}}^{2}\right) \otimes \cdots \otimes$ $\left(\sum f_{i_{k}}^{k}\right) \otimes\left(\sum f_{i_{1}}^{1}\right)$, where $f_{i_{1}}^{1}, \ldots, f_{i_{k}}^{k}: \Upsilon_{q} \rightarrow M$. Then $c=\sum_{i_{1}, \ldots, i_{k}} f_{i_{1}}^{1} \otimes$ $\cdots \otimes f_{i_{k}}^{k}$ and $c^{\prime}=\sum_{i_{1}, \ldots, i_{k}} f_{i_{2}}^{2} \otimes \cdots \otimes f_{i_{k}}^{k} \otimes f_{i_{1}}^{1}$ We see again that $\pi_{\#}(c+$ $\left.c^{\prime}\right)=0$. Similarly, $c_{1} \otimes c_{2} \otimes \cdots \otimes c_{k}+c_{k} \otimes \cdots \otimes c_{2} \otimes c_{1} \in \operatorname{ker} \pi_{\#} \cdot$

Suppose $c=f \otimes f \otimes f^{3} \otimes \cdots \otimes f^{k}$. Then $\pi_{\#}(c)$ maps the simplex $\Upsilon_{q}$ to the distinguished point, hence $\pi_{\#}(c)=0 \in K^{\prime}$. Now suppose $c=\left(\sum f_{i}\right) \otimes\left(\sum f_{i}\right) \otimes\left(\sum f_{i_{3}}^{3}\right) \otimes \cdots \otimes\left(\sum f_{i_{k}}^{k}\right)=\sum_{\substack{i_{1}, i_{2}, i_{3}, \ldots, i_{k} \\ i_{1} \leq i_{2}}}\left(f_{i_{1}}+f_{i_{2}}\right) \otimes$ $\left(f_{i_{2}}+f_{i_{1}}\right) \otimes f_{i_{3}}^{3} \otimes \cdots \otimes f_{i_{k}}^{k}$. We see that $\pi_{\#}(c)=0$.

Let us show that the FD-morphism $\alpha$ induces an isomorphism of homology groups. In the following lemma we construct a triangulation

\footnotetext{
${ }^{2}$ Notations $K(X)$ and $p t(X)$ were defined on page 34 .
} 
$\tau$ of the space $M^{\times k}$. The idea is as follows. This triangulation is coherent with the filtration

$$
\{(x, \ldots, x)\}=\Delta^{0} \subset \Delta_{M} \subset M^{\times k}
$$

and invariant under the action of the dihedral group $D_{k}$. Since $\tau$ is invariant under the action of $D_{k}, \tau$ induces a triangulation $\tau / D_{k}$ of the space $M^{\times k} / D_{k}$. Since the diagonal $\Delta=\Delta_{M}$ is triangulated, $\tau / D_{k}$ induces a cell decomposition $\pi(\tau)$ of the space $X$. All affine maps $f: \Upsilon_{q} \rightarrow M^{\times k}$ such that $f$ sends vertices of $\Upsilon_{q}$ to vertices of a simplex $\delta \in \tau$ constitute an FD-submodule $S \subset K$. The image $\pi_{\#}(S)=S^{\prime} \subset$ $K^{\prime}$ is an FD-submodule of $K^{\prime}$. By $S_{\text {sym }}$ we denote the intersection $K_{\text {sym }} \cap S$. There are inclusions $i: S_{\text {sym }} \rightarrow K_{\text {sym }}$ and $j: S \rightarrow K$, hence there is an inclusion $\tilde{j}: S / S_{\text {sym }} \rightarrow K / K_{\text {sym }}$. We show that these FD-modules and morphisms form the following commutative diagram with exact columns:

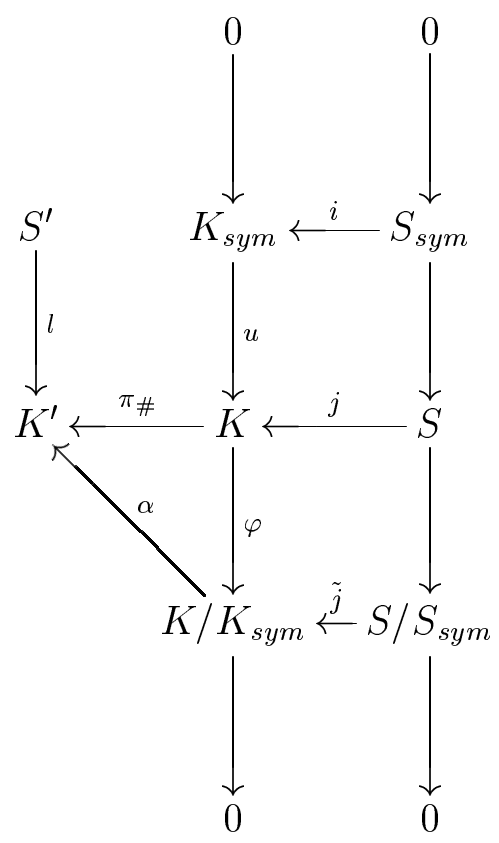

It is well known that the inclusions $j$ and $l$ induce isomorphisms of homology groups. It is easy to see that $i_{*}$ is an isomorphism too. Indeed, suppose we have a singular cycle $\zeta \in \operatorname{ker} \partial_{q} \subset\left(K_{\text {sym }}\right)_{q}$. Its 0skeleton consists of the points on the diagonal and pairs of symmetric 
points. Thus we can construct a homotopy $F_{t}$ of its 0 -skeleton such that the image of $F_{t}$ belongs to $K_{\text {sym }}$ for each $t$, and the image of $F_{1}$ belongs to $S_{\text {sym }}$. Then we can extend $F_{t}$ to 1 -skeleton, and so on. Now two short exact sequences of FD-modules give us two long sequences of homology groups. By the well known 5-lemma, it follows that $\tilde{j}_{*}$ is an isomorphism.

Now we compute ker $\pi_{\#}$. Suppose $c \in K, \pi_{\#}(c)=0$. Clearly,

$$
c=\sum_{m}\left(f_{1 m} \otimes \cdots \otimes f_{k m}+f_{1 m}^{\prime} \otimes \cdots \otimes f_{k m}^{\prime}\right)+\sum_{n} F_{n}
$$

where $F_{n}: \Upsilon_{q} \rightarrow \Delta$ are simplices in the diagonal, $\pi_{\#}\left(f_{1 m} \otimes \cdots \otimes f_{k m}\right)=$ $\pi_{\#}\left(f_{1 m}^{\prime} \otimes \cdots \otimes f_{k m}^{\prime}\right)$. It means that

$$
f_{1 m}, \ldots, f_{k m}, f_{1 m}^{\prime}, \ldots, f_{k m}^{\prime}: \Upsilon_{q} \rightarrow M
$$

and for each point $P \in \Upsilon_{q}$ there exists a permutation $\sigma \in D_{k}$ such that $\left(f_{1 m}^{\prime}(P), \ldots, f_{k m}^{\prime}(P)\right)=\left(f_{\sigma(1) m}(P), \ldots, f_{\sigma(k) m}(P)\right)$. Note that this permutation is the same for all $P \in \Upsilon_{q}$ if the image $\left(f_{1 m}^{\prime} \otimes \cdots \otimes f_{k m}^{\prime}\right)\left(\Upsilon_{q}\right)$ does not intersect the diagonal $\Delta$. If it does not, then $f_{1 m} \otimes \cdots \otimes f_{k m}+f_{1 m}^{\prime} \otimes \cdots \otimes f_{k m}^{\prime} \in \operatorname{ker} \varphi$.

Similarly, for each $F_{n}$ we have $F_{n}=g_{1 n} \otimes \cdots \otimes g_{k n}$, where

$$
g_{1 n}, \ldots, g_{k n}: \Upsilon_{q} \rightarrow M
$$

and for each $P \in \Upsilon_{q}$ there exists a pair of indices $i, j$ such that $g_{i n}(P)=$ $g_{j n}(P)$. If the image $F_{n}\left(\Upsilon_{q}\right)$ does not intersect the small diagonal $\Delta^{(0)}$, then this pair is the same for all $P \in \Upsilon_{q}$. As before, $F_{n} \in \operatorname{ker} \varphi$.

Now we see that all elements of ker $\alpha$ have either simplices whose images intersect the diagonal or simplices whose images lie in the diagonal and intersect the small diagonal. Clearly, it follows that $\operatorname{ker} \alpha \cap i m \tilde{j}=0$. Evidently, $\operatorname{im} \alpha \circ \tilde{j}=\mathrm{im} l$. We obtain that $\alpha \circ \tilde{j}: S / S_{\text {sym }} \rightarrow l\left(S^{\prime}\right)$ is an FD-isomorphism. We see that $l_{*}, \tilde{j}_{*}, l_{*}^{-1} \circ(\alpha \circ \tilde{j})_{*}$ are isomorphisms. Consequently, $\alpha_{*}=l_{*} \circ l_{*}^{-1} \circ(\alpha \circ \tilde{j})_{*} \circ \tilde{j}_{*}^{-1}$ is an isomorphism.

Lemma 3.2.2. Let $M$ be a polytope. As earlier, let

$$
\begin{gathered}
\Delta^{(0)}=\{(x, \ldots, x)\} \subset M^{\times k}, \\
\Delta=\left\{x_{1}=x_{2}\right\} \cup\left\{x_{2}=x_{3}\right\} \cup \cdots \cup\left\{x_{k-1}=x_{k}\right\} \cup\left\{x_{k}=x_{1}\right\} \subset M^{\times k} .
\end{gathered}
$$


Then there exists a triangulation $\tau$ of the space $M^{\times k}$ such that $\tau$ is coherent with the filtration

$$
\Delta^{(0)} \subset \Delta \subset M^{\times k}
$$

and invariant under the action of the dihedral group $D_{k}$ on $M^{\times k}$.

Proof. Since $M$ is triangulated, its Cartesian power $M^{\times k}$ is decomposed as follows

$$
M^{\times k}=\bigcup L_{i_{1}}^{q_{1}} \times \cdots \times L_{i_{k}}^{q_{k}},
$$

where $M=\bigcup L_{i}^{q}$ is the triangulation for the space $M$. Then we triangulate each $L_{i_{1}}^{q_{1}} \times \cdots \times L_{i_{k}}^{q_{k}}$ according to the next lemma and by the corollary 3.2.4 this triangulation is one we need.

Lemma 3.2.3. Let $\alpha$ be a fixed integer. Then for any ordered set $q_{1} \leq \cdots \leq q_{\alpha}$ of integers there exists a special triangulation $\tau_{q_{1} \leq \cdots \leq q_{\alpha}}$ of the Cartesian product $\Upsilon_{q_{1}} \times \cdots \times \Upsilon_{q_{\alpha}}$ such that the following conditions hold for the whole set of all special triangulations:

- They all are invariant under permutations of coordinates in any $\Upsilon_{q_{i}}$

- The special triangulation on the polyhedron $\Upsilon_{q_{1}} \times \cdots \times \Upsilon_{q_{\alpha}}$ induces the special triangulations on all its faces.

Proof. The proof is by induction over $\alpha$. First suppose $\alpha=2$. Let the simplices $\Upsilon_{1}$ and $\Upsilon_{2}$ be triangulized baricentrically, $U_{1}$ be the center of $\Upsilon_{1}, U_{2}$ be the center of $\Upsilon_{2}$. Then $U_{1} \times U_{2}$ is the center of the polyhedron $\Upsilon_{1} \times \Upsilon_{2}$. The triangulation we need is obtained from the triangulations for $\Upsilon_{1}$ and $\Upsilon_{2}$ by taking the cones over all the simplices with the vertex $U_{1} \times U_{2}$.

Suppose now we have proved the statement for some $\alpha$ and let us prove it for $\alpha+1$. By the inductive assumption the polyhedron $\Upsilon_{q_{1}} \times$ $\cdots \times \Upsilon_{q_{\alpha}}$ is already triangulated:

$$
\Upsilon_{q_{1}} \times \cdots \times \Upsilon_{q_{\alpha}}=\bigcup L_{i}^{q}
$$

We have the decomposition $\Upsilon_{q_{1}} \times \cdots \times \Upsilon_{q_{\alpha+1}}=\bigcup L_{i}^{q} \times \Upsilon_{q_{\alpha+1}}$ into the union of polyhedrons each of those can be triangulized according the inductive basis. The lemma is proved. 
Corollary 3.2.4. Let $\alpha \geq 2, q$ be integers. Then the special triangulation for $\Upsilon_{q}^{\times \alpha}$ constructed in the previous lemma satisfies the following conditions

1. It is invariant under the action of the dihedral group $D_{k}$ by permuting the direct factors (moreover, it is invariant under the action of the whole symmetric group $S_{k}$ ).

2. It is coherent with the filtration

$$
\Delta_{\Upsilon_{q}}^{(0)} \subset \Delta_{\Upsilon_{q}} \subset \Upsilon_{q}^{\times \alpha}
$$


Chapter 4

Lower estimates for the

number of closed billiard

trajectories 


\subsection{Proof of P. Pushkar's theorem}

Theorem 4.1.1. Let $M$ be a smooth closed connected $m$-dimensional manifold, $M \rightarrow \mathbb{R}^{n}$ a generic embedding, $B=\sum_{q=0}^{m} \operatorname{dim} H_{q}\left(M ; \mathbb{Z}_{2}\right)$. Then the number of 2-periodic billiard trajectories in $M$ is at least

$$
\frac{B^{2}+(m-1) B}{2}
$$

Proof. The statement follows from lemmas 3.2.1 and 4.1.3. Suppose $k_{i}=\operatorname{dim} H_{i}\left(M ; \mathbb{Z}_{2}\right)$. By $X$ denote the bouquet of spheres

$$
S^{m} \vee S_{1}^{m-1} \vee \cdots \vee S_{k_{m-1}}^{m-1} \vee \cdots \vee S_{1}^{1} \vee \cdots \vee S_{k_{1}}^{1}
$$

We see that the space $X$ has $\mathbb{Z}_{2}$-homology groups isomorphic to those of $M$. Using the results of Chapters 2 and 3, we get

$$
B T_{2}(M) \geq \sum_{i=0}^{2 m} \operatorname{dim} H_{i}\left(M^{\times 2} / D_{2}, \Delta ; \mathbb{Z}_{2}\right)=\sum_{i=1}^{2 m} \operatorname{dim} H_{i}\left(X^{\times 2} / D_{2}, \Delta_{X} ; \mathbb{Z}_{2}\right) .
$$

In the following lemmas we calculate the sum of Betti numbers

$$
\sum_{i=1}^{2 m} \operatorname{dim} H_{i}\left(X^{\times 2} / D_{2}, \Delta_{X} ; \mathbb{Z}_{2}\right)
$$

and find that it equals $\frac{B^{2}+(m-1) B}{2}$.

\section{Lemma 4.1.2.}

$$
\begin{gathered}
\sum \operatorname{dim} H_{q}\left(S^{m} \times S^{m} / \mathbb{Z}_{2}, \Delta ; \mathbb{Z}_{2}\right)=m+1, \\
H_{*}\left(S^{m} \times S^{m} / \mathbb{Z}_{2}, \Delta ; \mathbb{Z}_{2}\right)=\left\{0, \ldots, 0, \mathbb{Z}_{2}, \ldots, \mathbb{Z}_{2}\right\} .
\end{gathered}
$$

Proof. We construct a cell decomposition of the Cartesian square $S^{m} \times S^{m}$ such that this decomposition is invariant under the involution $(x, y) \rightarrow(y, x)$ and $\Delta_{S^{m}}=\{(x, x)\}$ is a cell subspace. We consider $S^{m}$ as $m$-dimensional cube $[0,1]^{\times m}$ with the boundary contracted to a point. So $S^{m} \times S^{m}$ is the Cartesian product of two such cubes. Let $\varphi_{1}, \ldots, \varphi_{m}$ be the coordinates on the first cube, $x_{1}, \ldots, x_{m}$ be the coordinates on the second cube. 
The cells are

$$
e_{i}^{q}=\left\{\varphi_{1} * x_{1}, \ldots, \varphi_{m} * x_{m}\right\},
$$

where $*$ is one of the signs $>,<,=$. Of course, not all of $*$ are $=$, since we have contracted the diagonal $\tilde{\Delta}$. The codimension of a cell is $\#(=)$. There are two $m$-dimensional cells:

$$
\begin{gathered}
e_{1}^{m}=\left\{\varphi_{1}=0, \ldots, \varphi_{m}=0\right\}, \\
e_{2}^{m}=\left\{x_{1}=0, \ldots, x_{m}=0\right\} .
\end{gathered}
$$

Evidently, they do not belong to the boundaries of $(m+1)$-dimensional cells.

This decomposition is invariant under the $D_{2}$-action, hence it induces a cell decomposition of the symmetric square $S^{m} \times S^{m} / D_{2}$ with a diagonal contracted to a point. Let us denote the corresponding chain complex by $C\left(S^{m} \times S^{m} / D_{2}, \Delta_{S^{m}}\right)$. Evidently, it equals the direct sum of two its subcomplexes. One of them is generated by all $e_{i}^{q}=\left\{\varphi_{1} * x_{1}, \ldots, \varphi_{m} * x_{m}\right\}$, let us denote it by $b\left(S^{m} \times S^{m} / D_{2}, \Delta_{S^{m}}\right)$, and another consists of one element $e^{m}=\left\{\varphi_{1}=0, \ldots, \varphi_{m}=0\right\}$, denote it by $s\left(S^{m} \times S^{m} / D_{2}, \Delta_{S^{m}}\right)$.

Obviously, $H_{m}\left(S^{m} \times S^{m} / \mathbb{Z}_{2}, \Delta\right) \cong \mathbb{Z}_{2}$ : there is only one cell of dimension $m$, its boundary is 0 .

Consider the Smith exact sequence of the Cartesian square $S^{m} \times S^{m}$ and the group $\mathbb{Z}_{2}$ acting on it (see [13]):

$$
\begin{gathered}
\ldots \longrightarrow H_{q}\left(S^{m} \times S^{m} / \mathbb{Z}_{2}, \Delta\right) \oplus H_{q}\left(S^{m}\right) \longrightarrow H_{q}\left(S^{m} \times S^{m}\right) \\
\longrightarrow H_{q}\left(S^{m} \times S^{m} / \mathbb{Z}_{2}, \Delta\right) \longrightarrow H_{q-1}\left(S^{m} \times S^{m} / \mathbb{Z}_{2}, \Delta\right) \oplus H_{q-1}\left(S^{m}\right) \longrightarrow \ldots
\end{gathered}
$$

Since $H_{q}\left(S^{m} \times S^{m}\right) \cong H_{q}\left(S^{m}\right) \cong 0$ if $q \neq 0, m, 2 m$, we have

$$
0 \longrightarrow H_{q}\left(S^{m} \times S^{m} / \mathbb{Z}_{2}, \Delta\right) \longrightarrow H_{q-1}\left(S^{m} \times S^{m} / \mathbb{Z}_{2}, \Delta\right) \longrightarrow 0
$$

where $q=m+2, \ldots, 2 m-1$.

Thus we see that $H_{m+1}\left(S^{m} \times S^{m} / \mathbb{Z}_{2}, \Delta\right) \cong \ldots \cong H_{2 m-1}\left(S^{m} \times\right.$ $\left.S^{m} / \mathbb{Z}_{2}, \Delta\right)$. 
Consider the end of the Smith sequence:

$$
\begin{gathered}
0 \longrightarrow H_{m+1}\left(S^{m} \times S^{m} / \mathbb{Z}_{2}, \Delta\right) \longrightarrow H_{m}\left(S^{m} \times S^{m} / \mathbb{Z}_{2}, \Delta\right) \oplus \mathbb{Z}_{2} \\
\longrightarrow \mathbb{Z}_{2} \oplus \mathbb{Z}_{2} \longrightarrow H_{m}\left(S^{m} \times S^{m} / \mathbb{Z}_{2}, \Delta\right) \longrightarrow 0 .
\end{gathered}
$$

Since $H_{m}\left(S^{m} \times S^{m} / \mathbb{Z}_{2}, \Delta\right) \cong \mathbb{Z}_{2}$, we see that $H_{m+1}\left(S^{m} \times S^{m} / \mathbb{Z}_{2}, \Delta\right) \cong$ $\mathbb{Z}_{2}$. Thus we have

$$
H_{m}\left(S^{m} \times S^{m} / \mathbb{Z}_{2}, \Delta\right) \cong \cdots \cong H_{2 m-1}\left(S^{m} \times S^{m} / \mathbb{Z}_{2}, \Delta\right) \cong \mathbb{Z}_{2} .
$$

Now consider the beginning of the Smith sequence:

$$
\begin{gathered}
0 \longrightarrow H_{2 m}\left(S^{m} \times S^{m} / \mathbb{Z}_{2}, \Delta\right) \longrightarrow \mathbb{Z}_{2} \longrightarrow H_{2 m}\left(S^{m} \times S^{m} / \mathbb{Z}_{2}, \Delta\right) \\
\longrightarrow H_{2 m-1}\left(S^{m} \times S^{m} / \mathbb{Z}_{2}, \Delta\right) \longrightarrow 0 .
\end{gathered}
$$

We already know that $H_{2 m-1}\left(S^{m} \times S^{m} / \mathbb{Z}_{2}, \Delta\right) \cong \mathbb{Z}_{2}$. Computing the Euler characteristic we obtain that $H_{2 m}\left(S^{m} \times S^{m} / \mathbb{Z}_{2}, \Delta\right) \cong \mathbb{Z}_{2}$. This completes the proof.

Lemma 4.1.3. Let $M$ be a smooth closed $m$-dimensional manifold, $k_{i}=\operatorname{dim} H_{i}\left(M ; \mathbb{Z}_{2}\right), i=0,1, \ldots, m, B=\sum_{i=0}^{m} k_{i}$. Then

$$
\sum_{i=1}^{m} i k_{i}=\frac{m B}{2}
$$

Proof. Using Poincaré duality, we have

$$
\begin{gathered}
\sum_{i=1}^{m} i k_{i}=\sum_{i=0}^{m} i k_{i}=\frac{1}{2} \sum_{i=0}^{m}\left(i k_{i}+(m-i) k_{m-i}\right) \\
=\frac{1}{2} \sum_{i=0}^{m}\left(i k_{i}+(m-i) k_{i}\right)=\frac{m B}{2} .
\end{gathered}
$$

Lemma 4.1.4. Let $M$ be a smooth closed connected m-dimensional manifold, $k_{i}=\operatorname{dim} H_{i}\left(M ; \mathbb{Z}_{2}\right), i=0,1, \ldots, m, B=\sum_{i=0}^{m} k_{i}$. By $X$ denote the bouquet of spheres

$$
S^{m} \vee S_{1}^{m-1} \vee \cdots \vee S_{k_{m-1}}^{m-1} \vee \cdots \vee S_{1}^{1} \vee \cdots \vee S_{k_{1}}^{1}
$$

Then we have

$$
\sum_{q=1}^{2 m} \operatorname{dim} H_{q}\left(X^{\times 2} / D_{2}, \Delta_{X} ; \mathbb{Z}_{2}\right)=\frac{B^{2}+(m-1) B}{2} .
$$


Proof. We construct a cell decomposition of the space $X^{\times 2} / D_{2}$ such that $\Delta_{X}=\{(x, x)\}$ is a cell subspace. The symmetric square $X^{\times 2} / D_{2}$ is the union of its subsets:

$$
X^{\times 2} / D_{2}=\bigcup_{\substack{1 \leq p \leq m \\ 1 \leq i \leq k_{p}}} A_{i}^{p} \cup \bigcup_{\substack{1 \leq p \leq m \\ 1 \leq i<j \leq k_{p}}} B_{i j}^{p} \cup \bigcup_{\substack{1 \leq p<q \leq m \\ 1 \leq i \leq k_{p}, 1 \leq j \leq k_{q}}} C_{i j}^{p q},
$$

where

- $A_{i}^{p}$ is the set of points $\left(x, x^{\prime}\right) \in X^{\times 2} / D_{2}$ such that $x, x^{\prime} \in S_{i}^{p}$. Thus $A_{i}^{p}$ is homeomorphic to $S^{p} \times S^{p} / D_{2}$.

- $B_{i j}^{p}$ is the set of points $\left(x_{1}, x_{2}\right) \in X^{\times 2} / D_{2}$ such that $x_{1} \in S_{i}^{p}$, $x_{2} \in S_{j}^{p}$. Thus $B_{i j}^{p}$ is homeomorphic to $S^{p} \times S^{p}$.

- $C_{i j}^{p q}$ is the set of points $(x, y) \in X^{\times 2} / D_{2}$ such that $x \in S_{i}^{p}, y \in S_{j}^{q}$. Thus $C_{i j}^{p q}$ is homeomorphic to $S^{p} \times S^{q}$.

For each $A_{i}^{p}$ we have constructed a cell decomposition earlier. For each $B_{i j}^{p}, C_{i j}^{p q}$ we have a standard decomposition:

$S^{p}=e^{0} \cup e^{p}, \quad S^{q}=e^{0} \cup e^{q}, \quad S^{p} \times S^{q}=e^{0} \times e^{0} \cup e^{0} \times e^{q} \cup e^{p} \times e^{0} \cup e^{p} \times e^{q}$

with all boundaries equal to 0 . By $s\left(B_{i j}^{p}\right)$ we denote a chain complex generated by one cell $e^{p} \times e^{p}$, by $s\left(C_{i j}^{p q}\right)$ we denote a chain complex generated by one cell $e^{p} \times e^{q}$.

Notice that these decompositions coincide on intersections of the sets $A_{i}^{p}, B_{i j}^{p}, C_{i j}^{p q}$.

Now we see that a chain complex for $R D^{2}(X)$ is a direct sum of its subcomplexes:

$$
\bigoplus_{\substack{1 \leq p \leq m \\ 1 \leq i \leq k_{p}}}\left(b\left(A_{i}^{p}\right) \oplus s\left(A_{i}^{p}\right)\right) \bigoplus_{\substack{1 \leq p \leq m \\ 1 \leq i<j \leq k_{p}}} s\left(B_{i j}^{p}\right) \bigoplus_{\substack{1 \leq p<q \leq m \\ 1 \leq i \leq k_{p}, 1 \leq j \leq k_{q}}} s\left(C_{i j}^{p q}\right)
$$

Now we compute contributions to the sum of Betti numbers 1.1 for each of these direct summands.

- $b\left(A_{i}^{p}\right) \oplus s\left(A_{i}^{p}\right)$ : The sum of Betti numbers of each $A_{i}^{p}$ is $p+1$. So we have a contribution $\sum_{i=1}^{m}(i+1) k_{i}$. 
Let us transform this expression using the previous lemma.

$$
\sum_{p=1}^{m}(p+1) k_{p}=B-1+\frac{m B}{2} .
$$

- $s\left(B_{i j}^{p}\right)$ : Each of $s\left(B_{i j}^{p}\right)$ contributes 1 to the sum of Betti numbers 1.1 , so totally we have

$$
\frac{1}{2} \sum_{p=1}^{m} k_{p}\left(k_{p}-1\right)
$$

- $s\left(C_{i j}^{p q}\right)$ :

$$
\sum_{p<q}^{m} k_{p} k_{q}
$$

Thus we have

$$
\begin{gathered}
\sum \operatorname{dim} H_{q}\left(X^{\times 2} / \mathbb{Z}_{2}, \Delta ; \mathbb{Z}_{2}\right)=B-1+\frac{m B}{2}+\frac{1}{2} \sum_{p=1}^{m} k_{p}\left(k_{p}-1\right)+\sum_{p<q}^{m} k_{p} k_{q} \\
=B-1+\frac{m B}{2}+\frac{1}{2}\left(\sum_{p=1}^{m} k_{p}\right)^{2}-\frac{1}{2} \sum_{p=1}^{m} k_{p} \\
=\frac{(B-1)^{2}+(m+1) B-1}{2}=\frac{B^{2}+(m-1) B}{2} .
\end{gathered}
$$

This completes the proof.

\subsection{Estimate for the number of 3-periodic trajectories}

Theorem 4.2.1. Let $M$ be a smooth closed connected m-dimensional manifold, $M \rightarrow \mathbb{R}^{n}$ a generic embedding. Suppose $k_{i}=\operatorname{dim} H_{i}\left(M ; \mathbb{Z}_{2}\right)$, $B=\sum_{i=0}^{m} k_{i}$. Then the number of 3-periodic billiard trajectories in $M$ is at least

$$
\frac{B^{3}+3(m-1) B^{2}+2 B}{6}
$$


Proof. As above, by the results of Section 2.2 and Section 3.2, it follows that

$B T_{3}(M) \geq \sum_{i=0}^{3 m} \operatorname{dim} H_{q}\left(M^{\times 3} / D_{3}, \Delta ; \mathbb{Z}_{2}\right)=\sum_{i=1}^{3 m} \operatorname{dim} H_{q}\left(X^{\times 3} / D_{3}, \Delta_{X} ; \mathbb{Z}_{2}\right)$,

where $X$ is a bouquet of spheres

$$
S^{m} \vee S_{1}^{m-1} \vee \cdots \vee S_{k_{m-1}}^{m-1} \vee \cdots \vee S_{1}^{1} \vee \cdots \vee S_{k_{1}}^{1}
$$

In the following lemmas we prove that

$$
\sum_{i=1}^{3 m} \operatorname{dim} H_{q}\left(X^{\times 3} / D_{3}, \Delta_{X} ; \mathbb{Z}_{2}\right)=\frac{B^{3}+3(m-1) B^{2}+2 B}{6} .
$$

Lemma 4.2.2. Let $M$ be $m$-dimensional manifold, $k_{i}=\operatorname{dim} H_{i}\left(M ; \mathbb{Z}_{2}\right)$, $i=0,1, \ldots, m, B=\sum_{i=0}^{m} k_{i}$. Then

$$
\sum_{1 \leq i \leq m} i k_{i}^{2}+\sum_{1 \leq i \neq j \leq m} i k_{i} k_{j}=\frac{m B^{2}-m B}{2} .
$$

Proof. Taking into account Poincaré duality $k_{i}=k_{m-i}$, we have

$$
\begin{gathered}
\sum_{1 \leq i \leq m} i k_{i}^{2}+\sum_{1 \leq i \neq j \leq m} i k_{i} k_{j}=\sum_{0 \leq i \leq m} i k_{i}^{2}+\sum_{0 \leq i \neq j \leq m} i k_{i} k_{j}-\sum_{1 \leq i \leq m, j=0} i k_{i} k_{j} \\
=\sum_{0 \leq i, j \leq m} i k_{i} k_{j}-\sum_{0 \leq i \leq m} i k_{i}= \\
=\frac{1}{2} \sum_{0 \leq i, j \leq m}(i+m-i) k_{i} k_{j}-\frac{1}{2} \sum_{0 \leq i \leq m}(i+m-i) k_{i} \\
=\frac{m B^{2}-m B}{2} .
\end{gathered}
$$

Lemma 4.2.3. Let $M$ be a smooth closed connected m-dimensional manifold, $k_{i}=\operatorname{dim} H_{i}\left(M ; \mathbb{Z}_{2}\right), B=\sum_{i=0}^{m} k_{i}$. Suppose

$$
X=S^{m} \vee S_{1}^{m-1} \vee \cdots \vee S_{k_{m-1}}^{m-1} \vee \cdots \vee S_{1}^{1} \vee \cdots \vee S_{k_{1}}^{1} \text {. }
$$

Then

$$
\sum_{i=1}^{3 m} \operatorname{dim} H_{i}\left(X^{\times 3} / D_{3}, \Delta_{X} ; \mathbb{Z}_{2}\right)=\frac{B^{3}+3(m-1) B^{2}+2 B}{6} .
$$


Proof. Let us construct a cell decomposition of the dihedral cube $X^{\times 3} / D_{3}$. The space $X^{\times 3} / D_{3}$ is a union of its subsets:

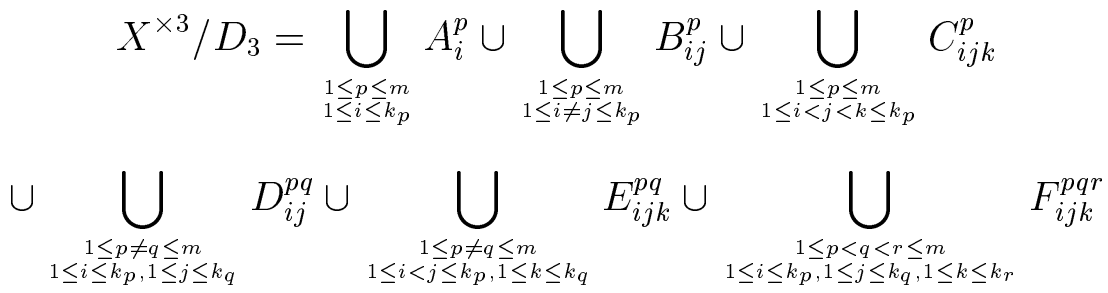

Here

- $A_{i}^{p}$ is the set of points $\left(x, x^{\prime}, x^{\prime \prime}\right) \in X^{\times 3} / D_{3}$ such that $x, x^{\prime}, x^{\prime \prime} \in S_{i}^{p}$. Thus it is homeomorphic to

$$
S^{p} \times S^{p} \times S^{p} / D_{3}
$$

- $B_{i j}^{p}$ is the set of points $\left(x_{1}, x_{1}^{\prime}, x_{2}\right) \in X^{\times 3} / D_{3}$ such that $x_{1}, x_{1}^{\prime} \in S_{i}^{p}$, $x_{2} \in S_{j}^{p}$. Thus it is homeomorphic to

$$
\left(S^{p} \times S^{p} / D_{2}\right) \times S^{p}
$$

- $C_{i j k}^{p}$ is the set of points $\left(x_{1}, x_{2}, x_{3}\right) \in X^{\times 3} / D_{3}$ such that $x_{1} \in S_{i}^{p}$, $x_{2} \in S_{j}^{p}, x_{3} \in S_{k}^{p}$. Thus it is homeomorphic to

$$
S^{p} \times S^{p} \times S^{p} .
$$

- $D_{i j}^{p q}$ is the set of points $\left(x, x^{\prime}, y\right) \in X^{\times 3} / D_{3}$ such that $x, x^{\prime} \in S_{i}^{p}$, $y \in S_{j}^{q}$. Thus it is homeomorphic to

$$
\left(S^{p} \times S^{p} / D_{2}\right) \times S^{q} .
$$

- $E_{i j k}^{p q}$ is the set of points $\left(x_{1}, x_{2}, y\right) \in X^{\times 3} / D_{3}$ such that $x_{1} \in S_{i}^{p}$, $x_{2} \in S_{j}^{p}, y \in S_{k}^{q}$. Thus it is homeomorphic to

$$
S^{p} \times S^{p} \times S^{q}
$$

- $F_{i j k}^{p q r}$ is the set of points $(x, y, z) \in X^{\times 3} / D_{3}$ such that $x \in S_{i}^{p}$, $y \in S_{j}^{q}, z \in S_{k}^{r}$. Thus it is homeomorphic to

$$
S^{p} \times S^{q} \times S^{r}
$$


Let us construct a cell decomposition for each $A_{i}^{p}=S^{p} \times S^{p} \times S^{p} / D_{3}$ like we have done it for $S^{p} \times S^{p} / D_{2}$. We consider a $p$-sphere as a $p$ dimensional cube $[0,1]^{\times p}$ with the boundary contracted to a point. Let $\varphi_{i}^{1}, \varphi_{j}^{2}, \varphi_{k}^{3}$ be the coordinates on three such cubes. There are the following cells. First

$$
U_{\beta}^{d}=\left\{\varphi_{1}^{i_{1}} * \varphi_{1}^{j_{1}} * \varphi_{1}^{k_{1}}, \ldots, \varphi_{m}^{i_{m}} * \varphi_{m}^{j_{m}} * \varphi_{m}^{k_{m}}\right\},
$$

where $*$ is one of the signs $<$ or $=,\left(i_{\alpha}, j_{\alpha}, k_{\alpha}\right)$ is a permutation of 1,2 , 3. Secondly

$$
V_{\beta}^{d}=\left\{\varphi_{1}^{1} * \varphi_{1}^{2}, \ldots, \varphi_{m}^{1} * \varphi_{m}^{2}, \vec{\varphi}^{3}=0\right\},
$$

where $*$ is one of the signs $<,>,=$. Clearly, the cells of the kind $U$ do not belong to the boundaries of the cells of the kind $V$. Similarly, the cells of the kind $V$ do not belong to the boundaries of the cells of the kind $U$. It is evident for $p>1$. For $p=1$ all boundary operators here vanish.

We see that a chain complex for $A_{i}^{p}$ is a direct sum of two subcomplexes $u\left(A_{i}^{p}\right)$ and $v\left(A_{i}^{p}\right)$.

Now we construct a cell decomposition for $B_{i j}^{p}$ and $D_{i j}^{p q}$. Here we have the same cells "of the kind $V$ ":

$$
V_{\beta}^{d}=\left\{\varphi_{1}^{1} * \varphi_{1}^{2}, \ldots, \varphi_{m}^{1} * \varphi_{m}^{2}, \vec{\psi}=0\right\}
$$

where $\vec{\varphi}^{1}$ and $\vec{\varphi}^{2}$ are the coordinates on $i$ th $p$-sphere, $\vec{\psi}$ are the coordinates on $j$ th $p$ - or $q$-sphere. Besides, there are the cells

$$
\begin{gathered}
W_{\beta}^{d}=\left\{\varphi_{1}^{1} * \varphi_{1}^{2}, \ldots, \varphi_{m}^{1} * \varphi_{m}^{2}, 0<x_{k}<1\right\}, \\
\tilde{W}_{\beta}^{d}=\left\{\vec{\varphi}^{1}=0,0<\varphi_{k}^{2}<1,0<x_{l}<1\right\} .
\end{gathered}
$$

As above, the cells of each of these three kinds form a chain subcomplex. Thus a chain complex for $B_{i j}^{p}$ is a direct $\operatorname{sum} v\left(B_{i j}^{p}\right) \oplus w\left(B_{i j}^{p}\right) \oplus$ $\tilde{w}\left(B_{i j}^{p}\right)$ and so is one for $D_{i j}^{p q}$.

Notice that

$$
\begin{gathered}
v\left(B_{i j}^{p}\right)=v\left(D_{i j}^{p q}\right)=v\left(A_{i}^{p}\right), \\
\tilde{w}\left(B_{i j}^{p}\right)=\tilde{w}\left(B_{j i}^{p}\right), \quad \tilde{w}\left(D_{i j}^{p q}\right)=\tilde{w}\left(D_{j i}^{q p}\right) .
\end{gathered}
$$

Clearly, each $\tilde{w}(\ldots)$ consists of only one cell with boundary equal to 0 . 
Finally, $C_{i j k}^{p}, E_{i j k}^{p q}$, and $F_{i j k}^{p q r}$ are Cartesian products of spheres, so here we have a standard cell decomposition:

$$
\begin{gathered}
S^{p}=e^{0} \cup e^{p}, \quad S^{q}=e^{0} \cup e^{q}, \quad S^{r}=e^{0} \cup e^{r}, \\
S^{p} \times S^{q} \times S^{r}=O \cup e^{0} \times e^{q} \times e^{r} \cup e^{p} \times e^{0} \times e^{r} \cup e^{p} \times e^{q} \times e^{0} \cup e^{p} \times e^{q} \times e^{r} .
\end{gathered}
$$

We see that $e^{0} \times e^{q} \times e^{r}, e^{p} \times e^{0} \times e^{r}$, and $e^{p} \times e^{q} \times e^{0}$ are cells "of the kind $\tilde{W}^{\prime \prime}$. The cell $e^{p} \times e^{q} \times e^{r}$ with vanishing boundary forms a chain subcomplex. We denote this subcomplex by $s\left(C_{i j k}^{p}\right), s\left(E_{i j k}^{p q}\right)$, or $s\left(F_{i j k}^{p q r}\right)$.

Now we see that the relative chain complex for the pair $\left(X^{\times 3} / D_{3}, \Delta_{X}\right)$ is a direct sum of subcomplexes:

$$
\bigoplus_{\substack{1 \leq p \leq m \\ 1 \leq i \leq k_{p}}}\left(u\left(A_{i}^{p}\right) \oplus v\left(A_{i}^{p}\right)\right) \bigoplus_{\substack{1 \leq p \leq m \\ 1 \leq i \neq j \leq k_{p}}} w\left(B_{i j}^{p}\right) \bigoplus_{\substack{1 \leq p \leq m \\ 1 \leq i<j \leq k_{p}}} \tilde{w}\left(B_{i j}^{p}\right) \bigoplus_{\substack{1 \leq p \leq m \\ 1 \leq i<j<k \leq k_{p}}} s\left(C_{i j k}^{p}\right)
$$

Now let us consider all these subcomplexes and compute their contributions to the sum of Betti numbers $\sum \operatorname{dim} H_{q}\left(X^{\times 3} / D_{3}, \Delta_{X} ; \mathbb{Z}_{2}\right)$.

- $u\left(A_{i}^{p}\right) \oplus v\left(A_{i}^{p}\right)$ : Each $A_{i}^{p}$ is a symmetric cube $S^{p} \times S^{p} \times S^{p} / D_{3}$. M. Farber and S. Tabachnikov in [7] and [8] proved that $\sum \operatorname{dim} H_{q}\left(S^{p} \times\right.$ $\left.S^{p} \times S^{p} / D_{3}, \Delta_{S_{p}} ; \mathbb{Z}_{2}\right)=2 p$, so the cell subcomplexes $A_{i}^{p}$ contribute $2 \sum_{p=1}^{m} p k_{p}=m B$.

Thus the contribution of the subcomplex under study is

$$
m B \text {. }
$$

- $w\left(B_{i j}^{p}\right): \sum_{p=1}^{m} p k_{p}\left(k_{p}-1\right)$. Indeed, for each $p$ we have $k_{p}\left(k_{p}-1\right)$ sets $B_{i j}^{p}$; the cells of the kind $W$ form the reduced dihedral square of the $p$-sphere without the unique $p$-dimensional cell that forms a subcomplex $\tilde{w}\left(B_{i j}^{p}\right)$. 
- $\tilde{w}\left(B_{i j}^{p}\right): \frac{1}{2} \sum_{p=1}^{m} k_{p}\left(k_{p}-1\right)$. Here $\frac{1}{2}$ appears, since $\tilde{w}\left(B_{i j}^{p}\right)=\tilde{w}\left(B_{j i}^{p}\right)$.

- $w\left(D_{i j}^{p q}\right): \sum_{p \neq q} p k_{p} k_{q}$.

- $\tilde{w}\left(D_{i j}^{p q}\right): \sum_{1 \leq p<q \leq m}^{m} k_{p} k_{q}$.

- $s\left(C_{i j k}^{p}\right)$ : Here we have subcomplexes consisting of one cell. Thus we have a contribution

$$
\frac{1}{6} \sum_{p=1}^{m} k_{p}\left(k_{p}-1\right)\left(k_{p}-2\right) \text {. }
$$

- $s\left(E_{i j k}^{p q}\right)$ : Here we have

$$
\frac{1}{2} \sum_{p \neq q}^{m} k_{p}\left(k_{p}-1\right) k_{q} .
$$

- $s\left(F_{i j k}^{p q r}\right)$ : Here we have

$$
\sum_{p<q<r}^{m} k_{p} k_{q} k_{r}
$$

Taking into account two computational lemmas, we have

$$
\begin{gathered}
m B+\sum_{p=1}^{m} p k_{p}\left(k_{p}-1\right)+\frac{1}{2} \sum_{p=1}^{m} k_{p}\left(k_{p}-1\right)+\sum_{p \neq q} p k_{p} k_{q}+\sum_{1 \leq p<q \leq m}^{m} k_{p} k_{q} \\
+\frac{1}{6} \sum_{p=1}^{m} k_{p}\left(k_{p}-1\right)\left(k_{p}-2\right)+\frac{1}{2} \sum_{p \neq q}^{m} k_{p}\left(k_{p}-1\right) k_{q}+\sum_{p<q<r}^{m} k_{p} k_{q} k_{r} \\
=m B+\frac{m B^{2}}{2}-\frac{m B}{2}-\frac{m B}{2}+\frac{(B-1)^{2}}{2}-\frac{B-1}{2}+\frac{(B-1)^{3}}{6}-\frac{(B-1)^{2}}{2}+\frac{B-1}{3} \\
=\frac{1}{6}\left(B^{3}-3 B^{2}+3 B-1+3 m B^{2}-B+1\right) \\
=\frac{B^{3}+3(m-1) B^{2}-2 B}{6} .
\end{gathered}
$$

This completes the proof. 


\subsection{Estimate for an arbitrary period}

Theorem 4.3.1. Let $M$ be a smooth closed connected m-dimensional manifold, $p$ an odd prime, $M \rightarrow \mathbb{R}^{n}$ a generic embedding. Suppose $k_{i}=\operatorname{dim} H_{i}\left(M ; \mathbb{Z}_{2}\right), B=\sum_{i=0}^{m} k_{i}$. Then the number of p-periodic billiard trajectories in $M$ is at least

$$
\frac{(B-1)\left((B-1)^{p-1}-1\right)}{2 p}+\frac{m B}{2}(p-1) .
$$

Proof. As above, by the results of Section 2.2 and Section 3.2, it follows that

$$
B T_{p}(M) \geq \sum_{i=0}^{p m} \operatorname{dim} H_{q}\left(M^{\times p} / D_{p}, \Delta ; \mathbb{Z}_{2}\right)=\sum_{i=0}^{p m} \operatorname{dim} H_{q}\left(X^{\times p} / D_{p}, \Delta_{X} ; \mathbb{Z}_{2}\right),
$$

where $X$ is a bouquet of spheres

$$
S^{m} \vee S_{1}^{m-1} \vee \cdots \vee S_{k_{m-1}}^{m-1} \vee \cdots \vee S_{1}^{1} \vee \cdots \vee S_{k_{1}}^{1}
$$

In the following lemma we prove that

$$
\sum_{i=1}^{p m} \operatorname{dim} H_{q}\left(X^{\times p} / D_{p}, \Delta_{X} ; \mathbb{Z}_{2}\right) \geq \frac{(B-1)\left((B-1)^{p-1}-1\right)}{2 p}+\frac{m B}{2}(p-1) .
$$

Lemma 4.3.2. Let $M$ be a smooth closed connected $m$-dimensional manifold, $p$ and odd prime, $k_{i}=\operatorname{dim} H_{i}\left(M ; \mathbb{Z}_{2}\right), B=\sum_{i=0}^{m} k_{i}$. Suppose

$$
X=S^{m} \vee S_{1}^{m-1} \vee \cdots \vee S_{k_{m-1}}^{m-1} \vee \cdots \vee S_{1}^{1} \vee \cdots \vee S_{k_{1}}^{1}
$$

Then

$\sum_{i=1}^{p m} \operatorname{dim} H_{i}\left(X^{\times p} / D_{p}, \Delta_{X} ; \mathbb{Z}_{2}\right) \geq \frac{(B-1)\left((B-1)^{p-1}-1\right)}{2 p}+\frac{m B}{2}(p-1)$.

Proof. Consider the bouquet of spheres $X$. By $X_{0}$ denote the common point of all the spheres. Let $X_{i}$ be the $i$ th sphere of the bouquet without the point $X_{0}$, so topologically $X_{i}$ is a Euclidean space $\mathbb{R}^{q}$ and $X=X_{0} \cup X_{1} \cup \cdots \cup X_{B-1}$ is a cell decomposition. 
Clearly we have

$$
X^{\times p}=\bigcup_{i_{1}, \ldots, i_{p}} X_{i_{1}} \times \cdots \times X_{i_{p}}
$$

is a cell decomposition of the Cartesian power $X^{p}$. What we do need is to construct its subdecomposition such that

- it is invariant under the action of the dihedral group $D_{p}$,

- the diagonal $\Delta$ is a cell subspace.

Note that if $i_{1} \neq i_{2} \neq i_{3} \neq \cdots \neq i_{p} \neq i_{1}$, then $X_{i_{1} \ldots i_{p}}=X_{i_{1}} \times \cdots \times X_{i_{p}}$ does not intersect the diagonal. It follows that $X_{i_{1} \ldots i_{p}}$ is a cell of the decomposition being constructed and its boundary is zero.

Consider now $X_{i_{1} \ldots i_{p}}=X_{i_{1}} \times \cdots \times X_{i_{p}}$ such that $i_{\alpha}=i_{\alpha+1}$ for some $\alpha \in \mathbb{Z}_{p}$. First suppose that not all of the $i_{\alpha}$ coincide. Without loss of generality we can assume that $i_{1}=\cdots=i_{\beta_{1}} \neq i_{\beta_{1}+1}=\cdots=i_{\beta_{2}} \neq$ $\cdots \neq i_{\beta_{u}+1}=\cdots=i_{p} \neq i_{1}$. We construct a cell subdecomposition for all $X_{i} \times X_{i} \times \cdots \times X_{i}$ and the decomposition for the whole $X_{i_{1} \ldots i_{p}}$ would be their tensor product.

Each $X_{i}$ is topologically a Euclidean space $\mathbb{R}^{q}$. Thus we deal with the Cartesian power $\left(\mathbb{R}^{q}\right)^{\times \beta}$. Let the $j$ th $\mathbb{R}^{q}$ have coordinates $x_{1}^{j}, \ldots, x_{q}^{j}$. A cell is given by the following conditions:

$$
\begin{array}{lllllll}
x_{1}^{1} & \varepsilon_{1}^{1} & x_{1}^{2} & \varepsilon_{1}^{2} & \ldots & \varepsilon_{1}^{\beta-1} & x_{1}^{\beta}, \\
x_{2}^{1} & \varepsilon_{2}^{1} & x_{2}^{2} & \varepsilon_{2}^{2} & \ldots & \varepsilon_{2}^{\beta-1} & x_{2}^{\beta}, \\
\vdots & & & & & & \\
x_{q}^{1} & \varepsilon_{q}^{1} & x_{q}^{2} & \varepsilon_{q}^{2} & \ldots & \varepsilon_{q}^{\beta-1} & x_{q}^{\beta} .
\end{array}
$$

where each $\varepsilon_{*}^{*}$ is one of the signs $<$, >, or $=$.

Now consider $X_{i_{1} \ldots i_{p}}$ having $i_{1}=\cdots=i_{p}=i$. Then the cell subdecomposition for this thing is given by the same construction with inequalities $x_{j}^{\beta} \varepsilon_{j}^{\beta} x_{j}^{1}$ added. Clearly, $\varepsilon_{j}^{1}, \varepsilon_{j}^{2}, \ldots, \varepsilon_{j}^{\beta}$ should not be all $<$ or all $>$, since in this case the system of inequalities has no solutions at all.

We have just constructed the cell decomposition for the space $X^{\times p}$. Denote the corresponding chain complex by $C\left(X^{\times p}\right)$. It induces the cell decomposition for the quotient $X^{\times p} / D_{p}$ with the diagonal contracted to 
a point. Let us denote the induced chain complex by $C\left(X^{\times p} / D_{p}, \Delta_{X}\right)$. Our goal is to calculate its homology

$$
H_{*} C\left(X^{\times p} / D_{p}, \Delta_{X}\right) .
$$

First consider $X_{i \ldots i}$ for some fixed $i>0$. Suppose $\operatorname{dim} X_{i}=q$. Note that all $X_{i_{1} \ldots i_{p}}$ such that $i_{\alpha}$ is either 0 or $i$ for all $\alpha=1, \ldots, p$ form a chain subcomplex. Denote it by $C^{+}\left(X_{i \ldots i}\right)$. Moreover, there is no cell outside $C^{+}\left(X_{i \ldots i}\right)$ such that its algebraic boundary contains terms lying in $C^{+}\left(X_{i \ldots i}\right)$. Hence $C^{+}\left(X_{i \ldots i}\right)$ is a direct summand in $C\left(X^{\times p} / D_{p}, \Delta_{X}\right)$. Obviously, $C^{+}\left(X_{i \ldots i}\right)$ coincides with a chain complex for a sphere $C\left(\left(S^{q}\right)^{\times p} / D_{p}, \Delta_{S^{q}}\right)$. By the results of M. Farber and S. Tabachnikov [7], it follows that it has the following sum of Betti numbers

$$
\sum \operatorname{dim} H_{\alpha}\left(C^{+}\left(X_{i \ldots i}\right) ; \mathbb{Z}_{2}\right)=q(p-1) .
$$

Summing for all $i$ and using Lemma 4.1.3, we obtain the following contribution to the sum of Betti numbers being calculated:

$$
\frac{m B}{2}(p-1)
$$

Now consider $X_{i_{1} \ldots i_{p}}=X_{i_{1}} \times \cdots \times X_{i_{p}}$ for $i_{1} \neq i_{2} \neq i_{3} \neq \cdots \neq i_{p} \neq i_{1}$. Each of these $X_{i_{1} \ldots i_{p}}$ is a cell such that

- its algebraic boundary is zero,

- it is not contained in an algebraic boundary of any other cell.

Hence it forms a chain subcomplex in $C\left(X^{\times p} / D_{p}, \Delta_{X}\right)$ consisting of only one group with only one generator and zero boundary operator. Let us denote this chain complex by $C\left(X_{i_{1} \ldots i_{p}}\right)$. It contributes 1 to the sum of Betti numbers being calculated.

It is well known from combinatorics that the number of all $X_{i_{1} \ldots i_{p}}$ having $i_{1} \neq i_{2} \neq i_{3} \neq \cdots \neq i_{p} \neq i_{1}$ equals

$$
(B-1)\left((B-1)^{p-1}-1\right) \text {. }
$$

Anyway let us prove it. Suppose $N(p)$ is the number of all $p$-tuples $\left(i_{1}, \ldots, i_{p}\right)$ such that $0 \leq i_{\alpha} \leq B-1$ and $i_{1} \neq i_{2} \neq i_{3} \neq \cdots \neq i_{p} \neq i_{1}$. Let now $p$ be not necessarily prime. Then we have

$$
N(p)=B(B-1)^{p-1}-N(p-1) .
$$


Indeed, $i_{1}$ may be chosen in $B$ ways. Each $i_{\alpha}, \alpha=2, \ldots, p$ may be chosen in $B-1$ ways to be different from $i_{\alpha-1}$. It gives $B(B-1)^{p-1}$. If we have got $i_{1}=i_{p}$, then $\left(i_{1}, i_{2}, \ldots, i_{p-1}\right)$ is a correct $(p-1)$-tuple. Thus recalling $p$ is an odd prime we get

$$
\begin{gathered}
N(p)=B(B-1)^{p-1}-N(p-1)=B(B-1)^{p-1}-B(B-1)^{p-2}+N(p-2)= \\
B(B-1)^{p-1}-B(B-1)^{p-2}+B(B-1)^{p-3}-\cdots-B(B-1)= \\
\cdots=B(1-B) \sum_{\alpha=0}^{p-2}(1-B)^{\alpha}= \\
B(1-B) \frac{1-(1-B)^{p-1}}{1-(1-B)}=(B-1)\left((B-1)^{p-1}-1\right) .
\end{gathered}
$$

This calculation gives us

$$
\frac{(B-1)\left((B-1)^{p-1}-1\right)}{2 p}
$$

when has factorized by the action of the dihedral group $D_{p}$. 
Chapter 5

Sharpness of given estimates 


\subsection{Plane billiards}

G. Birkhoff has found some lower estimate for the number of closed trajectories of a plane billiard. Nevertheless he has not proved that it is sharp.

Theorem 5.1.1. Suppose $p$ is an odd prime. There exists an embedding of the circle $S^{1}$ into the plane $\mathbb{R}^{2}$ such that the function

$$
l\left(x_{1}, \ldots, x_{p}\right)=\sum_{i \in \mathbb{Z}_{p}}\left\|x_{i}-x_{i+1}\right\|
$$

has $p-1$ critical points: $\frac{p-1}{2}$ maxima and $\frac{p-1}{2}$ points of Morse index $p-1$.

Proof. This embedding in polar coordinates is given by the formula

$$
r=1-\varepsilon \cos p \varphi
$$

for $\varepsilon$ small enough. Let us show this if $p=3$ (the proof for other values of $p$ is similar). 3-periodic billiard trajectories of the non-deformed circle $r=1$ are inscribed regular triangles. The coordinates of the vertices of such triangle are

$$
\varphi_{1}=\alpha_{0}, \varphi_{2}=\alpha_{0}+\frac{2 \pi}{3}, \varphi_{3}=\alpha_{0}+\frac{4 \pi}{3} .
$$

Thus 3-periodic billiard trajectories of the deformed circle $r=1-$ $\varepsilon \cos 3 \varphi$ are

$$
\varphi_{1}=\alpha_{0}+\beta_{1}, \varphi_{2}=\alpha_{0}+\beta_{2}+\frac{2 \pi}{3}, \varphi_{3}=\alpha_{0}+\beta_{3}+\frac{4 \pi}{3},
$$

where $\beta_{1}, \beta_{2}, \beta_{3} \rightarrow 0$ as $\varepsilon \rightarrow 0$. The length function is

$$
\begin{gathered}
f\left(\varphi_{1}, \varphi_{2}, \varphi_{3}\right)= \\
=\sqrt{\left(1-\varepsilon \cos 3 \varphi_{1}\right)^{2}+\left(1-\varepsilon \cos 3 \varphi_{2}\right)^{2}-2\left(1-\varepsilon \cos 3 \varphi_{1}\right)\left(1-\varepsilon \cos 3 \varphi_{2}\right) \cos \left(\varphi_{1}-\varphi_{2}\right)}+ \\
+\sqrt{\left(1-\varepsilon \cos 3 \varphi_{1}\right)^{2}+\left(1-\varepsilon \cos 3 \varphi_{3}\right)^{2}-2\left(1-\varepsilon \cos 3 \varphi_{1}\right)\left(1-\varepsilon \cos 3 \varphi_{3}\right) \cos \left(\varphi_{1}-\varphi_{3}\right)}+ \\
+\sqrt{\left(1-\varepsilon \cos 3 \varphi_{2}\right)^{2}+\left(1-\varepsilon \cos 3 \varphi_{3}\right)^{2}-2\left(1-\varepsilon \cos 3 \varphi_{2}\right)\left(1-\varepsilon \cos 3 \varphi_{3}\right) \cos \left(\varphi_{2}-\varphi_{3}\right)}
\end{gathered}
$$

Its derivatives have the following form:

$$
\frac{\partial}{\partial \varphi_{1}} f\left(\varphi_{1}, \varphi_{2}, \varphi_{3}\right)=
$$




$$
\begin{aligned}
= & \frac{3 \varepsilon\left(1-\varepsilon \cos 3 \varphi_{1}\right) \sin 3 \varphi_{1}-\left(1-\varepsilon \cos 3 \varphi_{2}\right)\left(3 \varepsilon \sin 3 \varphi_{1} \cos \left(\varphi_{1}-\varphi_{2}\right)-\left(1-\varepsilon \cos 3 \varphi_{1}\right) \sin \left(\varphi_{1}-\varphi_{2}\right)\right)}{\sqrt{\left(1-\varepsilon \cos 3 \varphi_{1}\right)^{2}+\left(1-\varepsilon \cos 3 \varphi_{2}\right)^{2}-2\left(1-\varepsilon \cos 3 \varphi_{1}\right)\left(1-\varepsilon \cos 3 \varphi_{2}\right) \cos \left(\varphi_{1}-\varphi_{2}\right)}}+ \\
& +\frac{3 \varepsilon\left(1-\varepsilon \cos 3 \varphi_{1}\right) \sin 3 \varphi_{1}-\left(1-\varepsilon \cos 3 \varphi_{3}\right)\left(3 \varepsilon \sin 3 \varphi_{1} \cos \left(\varphi_{1}-\varphi_{3}\right)-\left(1-\varepsilon \cos 3 \varphi_{1}\right) \sin \left(\varphi_{1}-\varphi_{3}\right)\right)}{\sqrt{\left(1-\varepsilon \cos 3 \varphi_{1}\right)^{2}+\left(1-\varepsilon \cos 3 \varphi_{3}\right)^{2}-2\left(1-\varepsilon \cos 3 \varphi_{1}\right)\left(1-\varepsilon \cos 3 \varphi_{3}\right) \cos \left(\varphi_{1}-\varphi_{3}\right)}} .
\end{aligned}
$$

Substituting $\varphi_{1}, \varphi_{2}$, and $\varphi_{3}$ for their values in this formula and the same formulas for $\frac{\partial}{\partial \varphi_{2}} f\left(\varphi_{1}, \varphi_{2}, \varphi_{3}\right)$ and $\frac{\partial}{\partial \varphi_{3}} f\left(\varphi_{1}, \varphi_{2}, \varphi_{3}\right)$, we obtain

$$
\begin{gathered}
\varphi_{1}=\alpha_{0}+\beta_{1}, \varphi_{2}=\alpha_{0}+\beta_{2}+\frac{2 \pi}{3}, \varphi_{3}=\alpha_{0}+\beta_{3}+\frac{4 \pi}{3}, \\
\cos 3 \varphi_{1}=\cos 3 \alpha_{0}-3 \beta_{1} \sin 3 \alpha_{0}+\ldots, \\
\cos 3 \varphi_{2}=\cos 3 \alpha_{0}-3 \beta_{2} \sin 3 \alpha_{0}+\ldots, \\
\cos 3 \varphi_{3}=\cos 3 \alpha_{0}-3 \beta_{3} \sin 3 \alpha_{0}+\ldots, \\
\sin 3 \varphi_{1}=\sin 3 \alpha_{0}+3 \beta_{1} \cos 3 \alpha_{0}+\ldots, \\
\sin 3 \varphi_{2}=\sin 3 \alpha_{0}+3 \beta_{2} \cos 3 \alpha_{0}+\ldots, \\
\sin 3 \varphi_{3}=\sin 3 \alpha_{0}+3 \beta_{3} \cos 3 \alpha_{0}+\ldots, \\
\cos \left(\varphi_{1}-\varphi_{2}\right)=-\frac{1}{2}+\frac{\sqrt{3}}{2}\left(\beta_{1}-\beta_{2}\right)+\ldots, \\
\cos \left(\varphi_{2}-\varphi_{3}\right)=-\frac{1}{2}+\frac{\sqrt{3}}{2}\left(\beta_{2}-\beta_{3}\right)+\ldots, \\
\cos \left(\varphi_{1}-\varphi_{3}\right)=-\frac{1}{2}-\frac{\sqrt{3}}{2}\left(\beta_{1}-\beta_{3}\right)+\ldots, \\
\sin \left(\varphi_{1}-\varphi_{2}\right)=-\frac{\sqrt{3}}{2}-\frac{1}{2}\left(\beta_{1}-\beta_{2}\right)+\ldots, \\
\sin \left(\varphi_{2}-\varphi_{3}\right)=-\frac{\sqrt{3}}{2}-\frac{1}{2}\left(\beta_{2}-\beta_{3}\right)+\ldots, \\
\sin \left(\varphi_{1}-\varphi_{3}\right)=\frac{\sqrt{3}}{2}-\frac{1}{2}\left(\beta_{1}-\beta_{3}\right)+\ldots
\end{gathered}
$$

Now we write that the derivatives of $f$ vanish:

$$
\begin{gathered}
-2 \sqrt{3} \sin 3 \alpha_{0}+\varepsilon \sqrt{3} \sin 3 \alpha_{0}+\beta_{1}\left(-6 \sqrt{3} \cos 3 \alpha_{0}-\frac{\sqrt{3}}{2}-\sin 3 \alpha_{0}\right)+ \\
+\beta_{2}\left(\frac{\sqrt{3}}{4}+\frac{1}{2} \sin 3 \alpha_{0}\right)+\beta_{3}\left(\frac{\sqrt{3}}{4}+\frac{1}{2} \sin 3 \alpha_{0}\right)+\cdots=0,
\end{gathered}
$$




$$
\begin{gathered}
-2 \sqrt{3} \sin 3 \alpha_{0}+\varepsilon \sqrt{3} \sin 3 \alpha_{0}+\beta_{1}\left(\frac{\sqrt{3}}{4}+\frac{1}{2} \sin 3 \alpha_{0}\right)+ \\
+\beta_{2}\left(-6 \sqrt{3} \cos 3 \alpha_{0}-\frac{\sqrt{3}}{2}-\sin 3 \alpha_{0}\right)+\beta_{3}\left(\frac{\sqrt{3}}{4}+\frac{1}{2} \sin 3 \alpha_{0}\right)+\cdots=0 \\
-2 \sqrt{3} \sin 3 \alpha_{0}+\varepsilon \sqrt{3} \sin 3 \alpha_{0}+\beta_{1}\left(\frac{\sqrt{3}}{4}+\frac{1}{2} \sin 3 \alpha_{0}\right)+ \\
+\beta_{2}\left(\frac{\sqrt{3}}{4}+\frac{1}{2} \sin 3 \alpha_{0}\right)+\beta_{3}\left(-6 \sqrt{3} \cos 3 \alpha_{0}-\frac{\sqrt{3}}{2}-\sin 3 \alpha_{0}\right)+\cdots=0 .
\end{gathered}
$$

Note that the constant term must be equal to 0 . Consequently $\alpha_{0}=0$ or $\alpha_{0}=\frac{\pi}{3}$. It is clear that $\beta_{1}=\beta_{2}=\beta_{3}=0$ is a periodic billiard trajectory for any $\varepsilon$, hence all coefficients of $\varepsilon^{k}$ are equal to 0 . Thus the dominant terms in this system are $\beta_{1}, \beta_{2}$, and $\beta_{3}$ with their coefficients. The linear system for $\beta_{1}, \beta_{2}, \beta_{3}$ has only the trivial solution. Hence we have the two trajectories: $\left(\frac{\pi}{3}, \pi, \frac{5 \pi}{3}\right)$ for the maximum and $\left(0, \frac{2 \pi}{3}, \frac{4 \pi}{3}\right)$ for the point of index 2 .

\subsection{Some conjecture on torus}

Suppose now we again have a smooth closed $m$-dimensional manifold $M$ embedded in Euclidean space $\mathbb{R}^{n}$. Given a prime integer $p$, we consider $p$-periodic billiard trajectories. We have just shown that for $m=1$ our estimates are sharp. Unfortunately, the only case except this one when there are some theorems about sharpness is $p=2$. Namely P. Pushkar in [6] has proved the following

Theorem 5.2.1. Suppose $M$ is a closed orientable 2-dimensional manifold, a sphere $S^{m}$, or a Cartesian product $S^{q} \times S^{r}$. Then there is a generic embedding $M \rightarrow \mathbb{R}^{n}$ for $n$ large enough such that the number of double normals equals

$$
\frac{B^{2}+(m-1) B}{2}
$$

where $B$ is the sum of Betti numbers and $m$ is the dimension of the manifold $M$.

In the previous section we have developed a method to construct examples with few enough closed billiard trajectories. Namely we have 
considered a regular circle $r=1$ and our examples were obtained by deformating the circle. It is not hard to see that a similar approach does not work when we deal with a torus in $\mathbb{R}^{3}$.

Indeed, consider a regular "round" torus

$$
\left\{\begin{array}{l}
x=(5+\cos \varphi) \cos \psi \\
y=(5+\cos \varphi) \sin \psi \\
z=\sin \varphi
\end{array}\right.
$$

Let us investigate closed billiard trajectories of period 3. They form some non-degenerate critical manifolds. Actually 3-periodic billiard trajectories are

- regular triangles inscribed in sections $\psi=$ const

- regular triangles inscribed in the circle $\varphi=0$

- regular triangles inscribed in the circle $\varphi=\pi$

- equilateral triangles with two vertices on $\varphi=0$ and one on $\varphi=\pi$

- equilateral triangles with two vertices on $\varphi=\pi$ and one on $\varphi=0$

- degenerate triangles with two vertices on $\varphi=0$ and one on $\varphi=\pi$

- degenerate triangles with two vertices on $\varphi=\pi$ and one on $\varphi=0$

- equilateral triangles with two vertices on $\psi=\psi_{0}$ and one $\psi=-\psi_{0}, \varphi=0$

- equilateral triangles with two vertices on $\psi=\psi_{0}$ and one $\psi=-\psi_{0}, \varphi=\pi$

We see here one critical torus and eight critical circles. The total Betti numbers sum of all of them equals 20 . The following statement holds:

Lemma 5.2.2. Let $N$ be a smooth manifold, $f: N \rightarrow \mathbb{R}$ a smooth function, $V \subset\{f=a\}$ a non-degenerate critical manifold. Suppose $\tilde{f}$ is a small deformation of the function $f$ such that $\tilde{f}$ and $\left.\tilde{f}\right|_{V}$ are Morse functions. Then $\tilde{f}$ has at least $\sum \operatorname{dim} H_{q}\left(V ; \mathbb{Z}_{2}\right)$ critical points. 
Proof. Suppose $\left.\tilde{f}\right|_{V}$ has $N$ critical points. Then $N \geq \sum \operatorname{dim} H_{q}\left(V ; \mathbb{Z}_{2}\right)$. A point $A \in V$ is critical for the function $\left.\tilde{f}\right|_{V}$ if $\operatorname{grad} \tilde{f} \perp T_{A} V$. In a neighborhood of a critical point we have

$$
\begin{gathered}
f=x_{1}^{2}+\cdots+x_{\alpha}^{2}-x_{\alpha+1}^{2}-x_{k}^{2}, \\
\tilde{f}=x_{1}^{2}+\cdots+x_{\alpha}^{2}-x_{\alpha+1}^{2}-x_{k}^{2}+\sum_{i=1}^{m} \varepsilon_{i} x_{i}+o(\mathbf{x}), \\
V=\left\{x_{k+1}=\cdots=x_{m}=0\right\} .
\end{gathered}
$$

Since $\operatorname{grad} \tilde{f} \perp V$, we have that $\varepsilon_{k+1}=\cdots=\varepsilon_{m}=0$. Hence the function $\tilde{f}$ has only one critical point in this neighborhood: $x_{i} \approx \frac{\varepsilon_{i}}{2}$. Thus the function $\tilde{f}$ has at least $N$ critical points.

Now we see that deformating the regular torus in $\mathbb{R}^{3}$ gives us at least 20 closed billiard trajectories of period 3 while the proved estimate is only 14 . Our conjecture is that if $S^{1} \times S^{1} \rightarrow \mathbb{R}^{3}$ is a generic embedding, then the number of 3-periodic billiard trajectories is at least 20. 


\section{Bibliography}

[1] G. Birkhoff.Dynamical systems. American Mathematical Sosciety. New York, 1927.

[2] M. Morse, S. S. Cairns. Critical points theory in global analysis and differential topology. Academic Press, New York, 1969.

[3] N .H. Kuiper. Double normals of convex bodies. Israel J. Math. 2(1964), 71-80.

[4] F. Takens, J. White. Morse theory of double normals of immersions. Indiana University Mathematics Journal, Vol. 21, No. 1, (1971), 11-17.

[5] I. Babenko. Periodic trajectories in three-dimensional billiards. Math. USSR Sbornik, no. 71 (1992), 1-13.

[6] P. Pushkar. Diameters of immersed manifolds and of wave fronts. C. R. Acad. Sci. 326 (1998), no. 2, 201-205.

[7] M. Farber, S. Tabachnikov. Topology of cyclic configuration spaces and periodic trajectories of multi-dimensional billiards. Topology 41 (2002), no. 3, 553-589.

[8] M. Farber, S. Tabachnikov. Periodic trajectories in 3dimensional convex billiards. Manuscr. Mat., 108 (2002), no. 4, 431-437.

[9] Fedor S. Duzhin. Lower bounds for the number of closed billiard trajectories of period 2 and 3 in manifolds embedded in Euclidean space. IMRN 2003, no. 8, 425-449. 
[10] V. I. Arnold Topological invariants of plane curves and caustics, Dean Jacqueline B. Lewis Memorial Lectures presented at Rutgers University, New Brunswick, New Jersey. University Lecture Series, 5. American Mathematical Society, Providence, RI, 1994.

[11] A. Dold. Homology of symmetric products and other functors of complexes. Annals of Mathematics, vol. 68, No. 1, July, 1958, 54-80.

[12] S. Eilenberg, S. MacLane. On the groups $H(\Pi, n)$. Annals of Mathematics, vol. 58, No. 1, July, 1953, 55-106.

[13] Glen E. Bredon. Introduction to compact transformation groups. Pure and Applied Mathematics, Vol. 46. Academic Press, New York-London, 1972.

[14] P. Griffiths, J. Harris. Principles of Algebraic Geometry. John Wiley \& Sons, 1978.

[15] A. Fomenko, D. Fuchs, V. Gutenmacher. Homotopic topology. Translated from the Russian by K. Mályusz. Akadémiai Kiadó (Publishing House of the Hungarian Academy of Sciences), Budapest, 1986.

[16] B. A. Dubrovin, A. T. Fomenko, S. P. Novikov. Modern geometry - methods and applications. Part I. The geometry of surfaces, transformation groups, and fields, 2nd ed., Graduate Texts in Mathematics, vol. 93, translated from the Russian by Robert G. Burns. Springer-Verlag, New York, 1992.

[17] B. A. Dubrovin, A. T. Fomenko, S. P. Novikov. Modern geometry - methods and applications. Part II. The geometry and topology of manifolds, Graduate Texts in Mathematics, vol. 104, translated from the Russian by Robert G. Burns. Springer-Verlag, New York, 1985.

[18] B. A. Dubrovin, A. T. Fomenko, S. P. Novikov. Modern geometry - methods and applications. Part III. Introduction to homology theory, Graduate Texts in Mathematics, vol. 124, translated from the Russian by Robert G. Burns. SpringerVerlag, New York, 1990. 